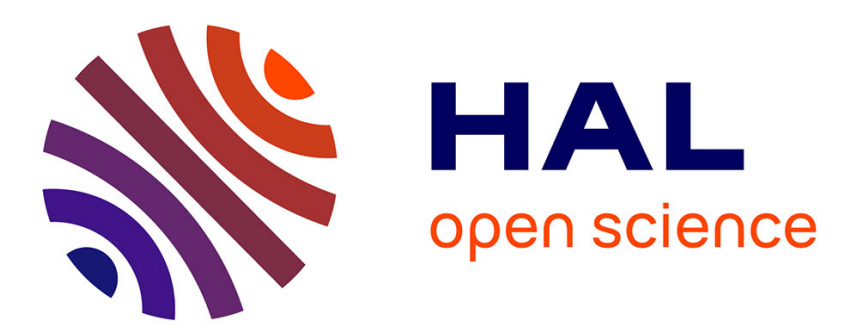

\title{
L'évolution ralentie du milieu naturel dans la steppe aride du nord de la Syrie à l'Holocène
}

\author{
Jean-Baptiste Rigot
}

\section{To cite this version:}

Jean-Baptiste Rigot. L'évolution ralentie du milieu naturel dans la steppe aride du nord de la Syrie à l'Holocène. Géomorphologie: relief, processus, environnement, 2006, 4, pp.259-274. hal-00943464

\section{HAL Id: hal-00943464 \\ https://hal.science/hal-00943464}

Submitted on 7 Feb 2014

HAL is a multi-disciplinary open access archive for the deposit and dissemination of scientific research documents, whether they are published or not. The documents may come from teaching and research institutions in France or abroad, or from public or private research centers.
L'archive ouverte pluridisciplinaire HAL, est destinée au dépôt et à la diffusion de documents scientifiques de niveau recherche, publiés ou non, émanant des établissements d'enseignement et de recherche français ou étrangers, des laboratoires publics ou privés. 

processus, environnement

4/2006 (2007)

Varia

Jean-Baptiste Rigot

\title{
L'évolution ralentie du milieu naturel dans la steppe aride du nord de la Syrie à I'Holocène
}

\author{
Avertissement \\ Le contenu de ce site relève de la législation française sur la propriété intellectuelle et est la propriété exclusive de \\ l'éditeur. \\ Les œuvres figurant sur ce site peuvent être consultées et reproduites sur un support papier ou numérique sous \\ réserve qu'elles soient strictement réservées à un usage soit personnel, soit scientifique ou pédagogique excluant \\ toute exploitation commerciale. La reproduction devra obligatoirement mentionner l'éditeur, le nom de la revue, \\ l'auteur et la référence du document. \\ Toute autre reproduction est interdite sauf accord préalable de l'éditeur, en dehors des cas prévus par la législation \\ en vigueur en France.
}

\section{revues.org}

Revues.org est un portail de revues en sciences humaines et sociales développé par le Cléo, Centre pour l'édition électronique ouverte (CNRS, EHESS, UP, UAPV).

\footnotetext{
Référence électronique

Éditeur : Groupe français de géomorphologie http://geomorphologie.revues.org http://www.revues.org

Document accessible en ligne sur : http://geomorphologie.revues.org/65 Ce document est le fac-similé de l'édition papier.

( ) Groupe français de géomorphologie
}

Jean-Baptiste Rigot, «L'évolution ralentie du milieu naturel dans la steppe aride du nord de la Syrie à l'Holocène », Géomorphologie : relief, processus, environnement [En ligne], 4/2006 | 2007, mis en ligne le 01 janvier 2009, consulté le 12 octobre 2012. URL : http://geomorphologie.revues.org/65 ; DOI : 10.4000/geomorphologie.65 


\title{
L'évolution ralentie du milieu naturel dans la steppe aride du nord de la Syrie à l'Holocène Slow rates of change in the natural arid steppe landscape
of northern Syria during the Holocene
}

\author{
Jean-Baptiste Rigot*
}

\section{Résumé}

L'étude géomorphologique de la région du lac Jabbûl, en Syrie du Nord, révèle que la dynamique morphogénétique à l'Holocène moyen et supérieur est faible. Pourtant, un épisode de morphogenèse important s'est déroulé au début de l'Holocène, que l'on peut rattacher à la phase climatique appelée ici «Optimum climatique holocène » (entre 9000 et 7000 ans BP environ). Par la suite, plusieurs petites phases morphogénétiques se manifestent. Elles seraient contemporaines du Bronze moyen (2 100 à 1600 av. J.-C.) et des époques romaine (64 av. J.-C. à $395 \mathrm{AD})$ et byzantine (395 à $636 \mathrm{AD})$. Cette morphogenèse relativement modeste se manifeste par le dépôt de nappes alluviales grossières formant des basses terrasses parfois épaisses mais limitées à des chenaux étroits. On constate que le fort alluvionnement des vallées, consécutif à une intense érosion des couvertures pédologiques, largement observé dans le bassin méditerranéen entre l'époque romaine et le Haut Moyen Âge, est inconnu dans la région étudiée. La disposition orographique en Syrie du nord, responsable de la faiblesse des précipitations, la lithologie et l'intense aménagement des versants aux époques romaine et byzantine, ont contribué à freiner le décapage des sols en limitant l'érosion des versants.

Mots clés : Holocène, morphogenèse, stabilité, aridité, steppe, Syrie.

\begin{abstract}
This geomorphological study of the Jabbûl lake region shows that landscape change was very slow during middle and late Holocene times. An intense erosional episode occurred at the beginning of the Holocene associated with the climatic phase known as "Holocene climatic optimum" (between 9000 yr BP and 7000 yr BP). It was followed by a decrease in morphogenic activity. Brief erosional episodes followed during the Middle Bronze (2100 to 1600 BC), Roman (64 BC to 395 AD) and Byzantine (395 to 636 AD) periods. These lower intensity episodes generated pebble terraces that are sometimes thick but in each case occupy a very narrow belt in river channels. Furthermore, the general phenomenon of soil erosion observed in the Mediterranean basin, from the Roman period to the early Middle Ages, is unknown here. Reasons given for this are the pattern of mountain topography in northern Syria, which explains the limited rainfall, and the widespread occurrence of resistant bedrock. However, extensive agricultural slope terracing during the Roman and Byzantine periods also assisted in mitigating soil erosion.
\end{abstract}

Key words: Holocene, erosion, stability, aridity, steppe, Syria.

\section{Abridged English Version}

This basin contains a salt lake in its centre, surrounded by two plateaux, the Jabal al-Has to the west and the Jabal Shbayth to the south. The climate is a degraded Mediterranean type characterized by winter rainfall and hot dry summers. Mean rainfall is very irregularly distributed, ranging from $300 \mathrm{~mm}$ in the northwest, to $193 \mathrm{~mm}$ in the southeast (fig. 2 and 3). The mean annual number of rainy days is 50. The mean annual temperature is $17^{\circ} \mathrm{C}$, includ- ing large differences between winter (minimum $5{ }^{\circ} \mathrm{C}$ in January) and summer (maximum over $30{ }^{\circ} \mathrm{C}$ from June to September). Strong westerly winds blow during spring and summer. These characteristics typify an arid environment in which mechanical erosion prevails.

The Aleppo region is a large structural unit called the "Aleppo platform" (Ponikarov et al. 1966). The Jabbûl lake area is located in the centre of this unit. Paleogene and Neogene rocks (fig. 4) are essentially calcareous, basaltic and conglomeratic. Pleistocene deposits are characterized

\footnotetext{
* Université de Tours, Laboratoire Archéologie et Territoires, CITERES-UMR 6173, 35 allée Ferdinand-de-Lesseps, BP 60449, 37204 Tours cedex

03. Courriel : jb.rigot@univ-tours.fr
} 
by detritic calcareous sediments in the basin, with gypsum layers.

The plateaus are covered by basaltic lavas. Jabal al-Has culminates at $650 \mathrm{~m}$ and Jabal Shbayth at $470 \mathrm{~m}$. Their slopes are cut by small, deep valleys which have recorded the recent geomorphic history of the region. Two series of fill terraces can be observed, where the lower terrace is set within the upper one. An upper terrace located in the Jabal Shbayht (fig. 5, section 4) shows a silty-sandy deposit at its base, overlain by a thin layer of sand and gravels, a deposit of silt and sand mixed with gravels and pebbles, and a siltysandy soil at the top. This terrace contains Neolithic artifacts (probably of Pre Pottery Neolithic B, dating to 9.2 ka to 8 ka year BP). The lower terrace (fig. 5, section 6) shows a silty-sandy palaeosol with a few small pebbles at the base, overlain by a deposit of gravels, pebbles and cobbles in a silty matrix, and a silty-sandy soil at the top. This terrace contains Roman artifacts (64 BC to 395 AD). Another post-Roman pebble terrace can be observed in the Jabal al-Has plateau (fig. 6B and fig. 9, section 7). These deposit are observed in other places on the plateaux but the chronology may be different: the upper terrace seems older at the base (Pre Pottery Neolithic A, 10 ka to 9 ka year BP) (fig. 5, section 1 and fig. 6A), whereas the lower one ranges from the middle Bronze Age (2100 BC to 1600 BC) (fig. 5, section 5) to the Byzantine period (between $395 A D$ and 636 AD) (fig. 7 and fig. 9, section 8)

These two series of terraces reflect at least two phases of geomorphic change (fig. 7 and fig 10). The first shows two deposits (QOa) characterized by pebbles and sand at the base covered by a sandy-silty layer. The basal deposit provides evidence of a flood, while the upper one corresponds to a weaker morphogenetic phase, in a less arid climate. The Neolithic artefacts found in this terrace (from 10000 to 8000 yr BP) confirm that the sediment was deposited during the relatively humid Holocene climatic optimum (fig. 10) (Sanlaville, 1996; Ayalon et al., 1999; RossignolStrick, 1999; Bar-Matthews et al., 2000). The influence of the Holocene climatic optimum on landscape evolution has also been noted in the Euphrates valley (Geyer and Besançon, 1997), in the Sajour Valley in northern Syria (Besançon and Sanlaville, 1985) and in the Khabur valley (Courty, 1994).

The second series of terraces $(Q 0 b)$ consists of pebble and sand with some silty layers. Only one of the terraces contains Bronze Age artefacts, while a few others contain Roman or Byzantine artefacts. The deposits are up to $4 \mathrm{~m}$ thick (fig. 9, section 8) but are confined to a very narrow channel. The alluviation and flooding mechanism is complex, but it cannot be argued on the basis of the evidence that the Jabbûl lake region experienced, at least until the beginning of the Roman period, the short climatic optimum (fig. 10) reported from neighbouring regions such as the Euphrates (Geyer and Besançon, 1997), Sajour (Besançon and Sanlaville, 1985) and Balikh valleys (Wilkinson, 1999). It has also been noted in the Mediterranean basin and in the Middle East, where the terraces are described as thick, wide and silt-dominated. They are thought to be induced partly by human impacts and partly by climatic change, i.e. the intense agricultural activity associated with the climatic optimum during the Hellenistic and Roman periods has accelerated runoff and soil erosion (Vita-Finzi, 1969; Dufaure, 1984; Fouache, 1999). The lower terraces in the Jabbûl lake region belong to this morphogenetic episode, but are smaller, less silty and do not suggest the intense soil erosion reported elsewhere. This comparatively limited erosional crisis can be explained by the influence of the Syrian coastal and Taurus Mountains, which isolate the region of Lake Jabbûl from the Mediterranean and from northern climatic influences. Furthermore, the resistant basaltic caprock minimises slope erosion on the plateaux. Finally, evidence of more intense erosion where the slopes are not covered by cultural terraces indicates that agricultural terracing of the plateau slopes during the Roman and Byzantine periods also helped to mitigate soil erosion.

In conclusion, the environmental changes around the Mediterranean basin during the Roman climatic optimum did not occur with the same intensity in the Jabbûl lake region as elsewhere. The orographic configuration of northern Syria separates the continental steppe regions from the Mediterranean zone. Consequently, this semi-arid area should not be considered as part of the Mediterranean region in terms Holocene erosional signatures.

\section{Introduction}

L'évolution morphologique de la Syrie continentale et plus encore celle des marges arides du nord de la Syrie à l'Holocène est encore assez mal connue malgré les synthèses de Butzer (1995), Sanlaville (1996 et 1997), Geyer (1999) et Wilkinson (1999) sur la Syrie et le Proche-Orient. Les données que nous possédons restent fragmentaires, particulièrement pour la période des 5000 dernières années. Cette contribution se propose d'apporter de nouveaux éléments de compréhension de la morphodynamique et des différentes phases de morphogenèse à l'Holocène sur les marges arides du Levant nord. L'étude est fondée sur une analyse géomorphologique détaillée de la région du lac Jabbûl, située dans la steppe aride du nord de la Syrie, avec des précipitations annuelles moyennes situées entre $100 \mathrm{~mm}$ et $400 \mathrm{~mm}$. Cette région déprimée, particulièrement favorable au piégeage de marqueurs morphosédimentaires, a gardé la trace d'une phase d'alluvionnement notable à l'Holocène ancien. En revanche, malgré des conditions idéales de sédimentation, les témoignages d'une activité morphologique postérieure sont rares et de faible envergure. Une longue accalmie morphologique a perduré jusqu'à l'époque contemporaine. Ce résultat est paradoxal au regard des épisodes de forte pression humaine et agricole que la région a connus, en particulier lors des époques romaine et byzantine. Il contraste également avec ce qui se passe au même moment sur le pourtour méditerranéen. L'analyse globale du milieu permet de replacer la région du lac Jabbûl dans un contexte naturel et humain qui appartient bien davantage au monde aride qu'au monde méditerranéen, expliquant ainsi cet apparent paradoxe. 


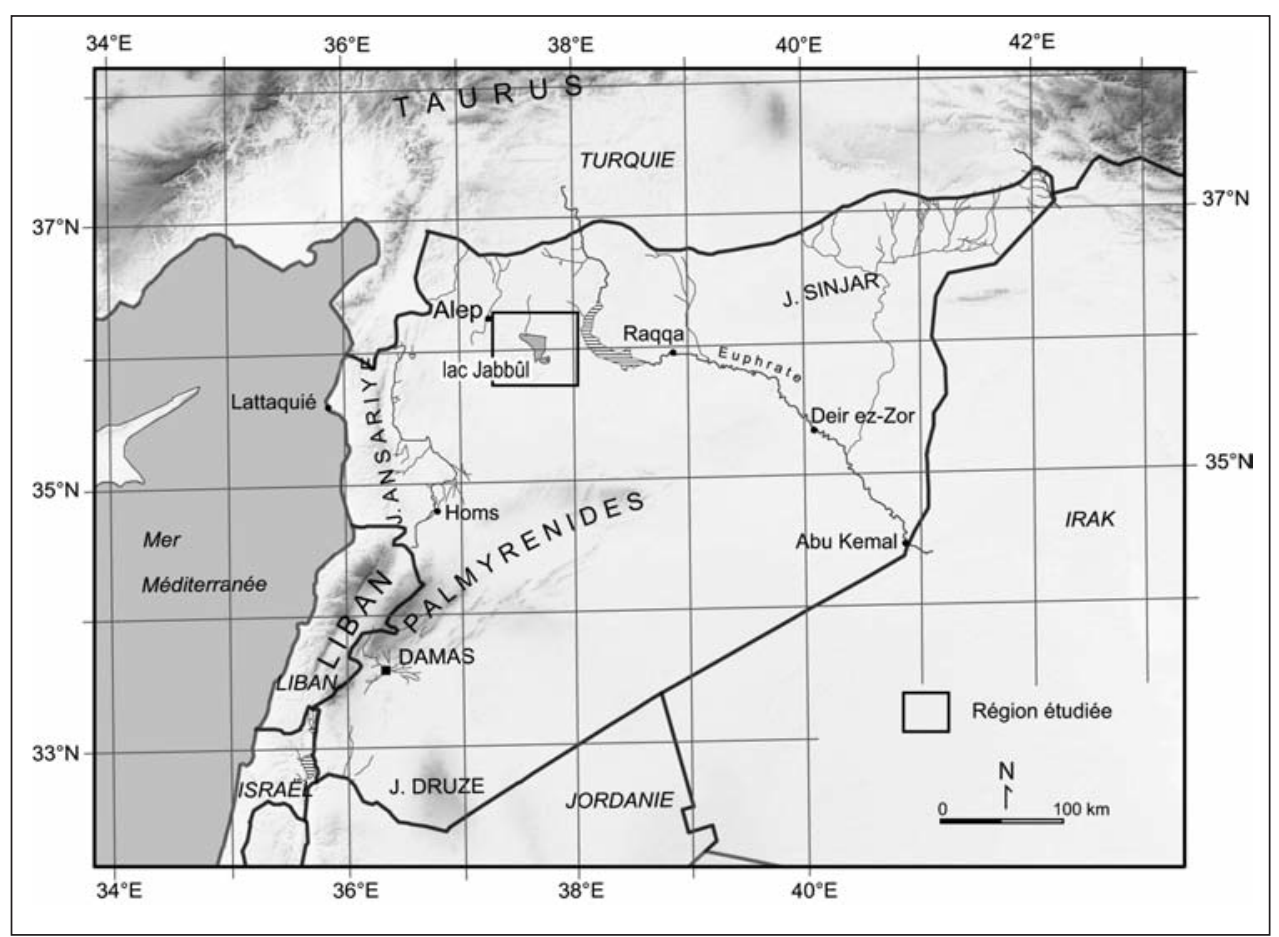

Fig. 1 - Syrie et région d'étude.

Fig. 1 - Study area in Syria.

de sa dynamique pluviométrique (pluies d'orages, courtes et brutales) et de sa forte aridité. L'orographie, Jabal Ansariyé et Taurus, accentue cette aridité et la dégradation du climat vers l'est et le sud en bloquant les dépressions provenant de la Méditerranée et du nord (Leroux, 1993, 1996 ; Traboulsi, 1981) (fig. 1). En position d'abri, la région reçoit $300 \mathrm{~mm}$ de précipitations à Sfirat au nord-ouest du secteur étudié, mais uniquement 193 mm à Khanasir au sud, alors que $813 \mathrm{~mm}$ tombent annuellement sur Lattaquié

\section{La région du lac Jabbûl : contexte actuel de la morphogenèse}

La région du lac Jabbûl, d'une superficie d'environ $3000 \mathrm{~km}^{2}$, est située au nord de la Syrie, à une cinquantaine de kilomètres au sud-est d'Alep (fig. 1). Elle est centrée sur le lac Jabbûl, un lac temporaire salé, bordé par deux mesas basaltiques à l'ouest et au sud et par des aplanissements au nord et au nord-ouest. Cette étude s'intéresse surtout aux piémonts et aux vallées issues de ces mesas, qui fournissent l'essentiel des informations morphologiques.

\section{Climat et morphogenèse}

Le climat de la région est de type méditerranéen dégradé (Traboulsi, 1981), en raison de son régime pluviothermique (pluies d'hiver et de printemps, sécheresse d'été),

Fig. 2 - Répartition des précipitations moyennes annuelles en Syrie et au Liban (19601961 à 1989-1990). D'après Traboulsi et Laborde (2001).

Fig. 2 - Distribution of mean annual rainfall in Syria and Lebanon (1960-1961 to 19891990), after Traboulsi and. Laborde (2001). sur la côte (fig. 2). Par ailleurs, les pluies se concentrent sur un temps court : environ 50 jours par an, tandis que le mois le plus arrosé représente $20 \%$ des précipitations annuelles. Enfin, ces précipitations sont très irrégulières, de $350 \mathrm{~mm}$ de précipitations sur l'ensemble de la région pour une année humide à $100 \mathrm{~mm}$ pour une année sèche (fig. 3). Les températures moyennes annuelles montrent une nette opposition entre un hiver rude et un été très chaud. La température moyenne est inférieure à $10^{\circ} \mathrm{C}$ de décembre à février. Les

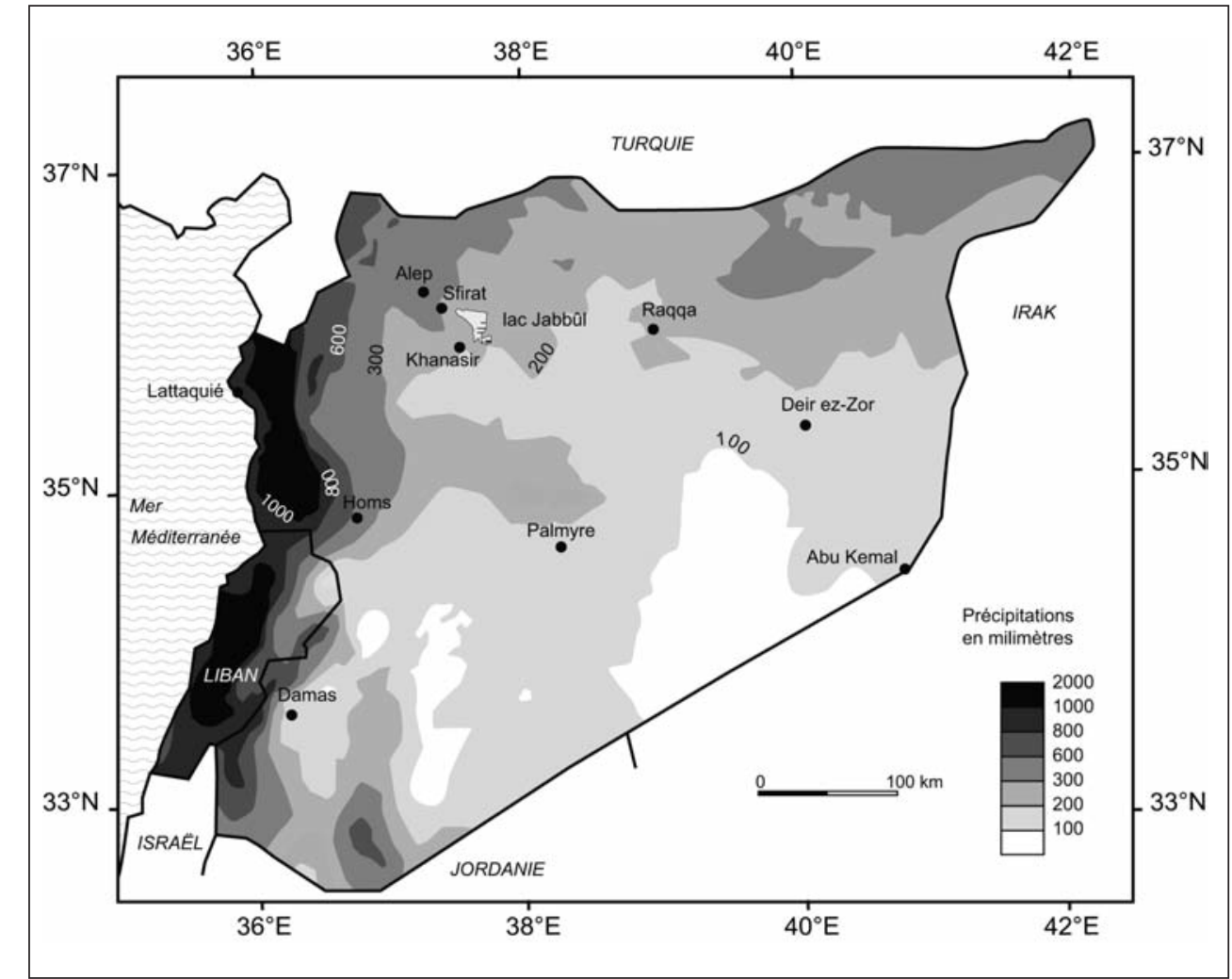


Fig. 3 - Précipitations en année humide et en année sèche en Syrie. A : les précipitations en année humide : l'exemple de l'année 1966-1967 ; B : les précipitations en année sèche : l'exemple de l'année 1972-1973 (d'après Traboulsi, 2004).

Fig. 3 - Rainfall for wet and dry years in Syria. A: Wet year precipitation: the example of 1966-1967; $B$ : Dry year precipitation: the example of 1972-1973 (after Traboulsi, 2004).

jours de gel sont fréquents : 25 jours par an en moyenne à Alep. En été, les températures maximales journalières sont supérieures à $30^{\circ} \mathrm{C}$. Le potentiel d'évapotranspiration (ETP) est élevé : les calculs effectués par Deiri (1990), à partir de relevés réalisés à Khanasir entre 1957 et 1986, livrent une moyenne de $2100 \mathrm{~mm}$ par an. Les grandes étendues planes et dénudées, qui constituent l'un des traits caractéristiques du paysage régional, favorisent une instabilité de l'air génératrice de vents puissants et d'une forte évaporation. L'activité éolienne se manifeste toute l'année, mais c'est en été et en hiver que les vents sont les plus forts. Ainsi, la distribution temporelle et la dynamique des précipitations, tout comme les forts contrastes de température et la persistance de l'activité éolienne conditionnent l'évolution actuelle du modelé. Cette évolution se manifeste par le ruissellement diffus, l'alluvionnement grossier en fond d'oued et l'érosion des sols poudreux par l'action éolienne. La fragilisation des roches par les gelées d'hiver et d'automne, l'action des sels hygroscopiques sur le basalte et la thermoclastie fournissent le matériau qui est transporté et déposé. L'érosion éolienne des sols est un phénomène de rétroaction positive consécutif à une extension irraisonnée des cultures en zone aride, impliquant l'arrachage de la végétation pérenne suivi des labours sur un sol limoneux. Le taux de recouvrement de la végétation dans les secteurs pâturés ne dépasse pas $20 \%$, sauf dans les cuvettes plus humides (Jaubert et al., 1999). D'après nos observations, ce mode d'érosion des sols s'est surtout imposé au XXe siècle.

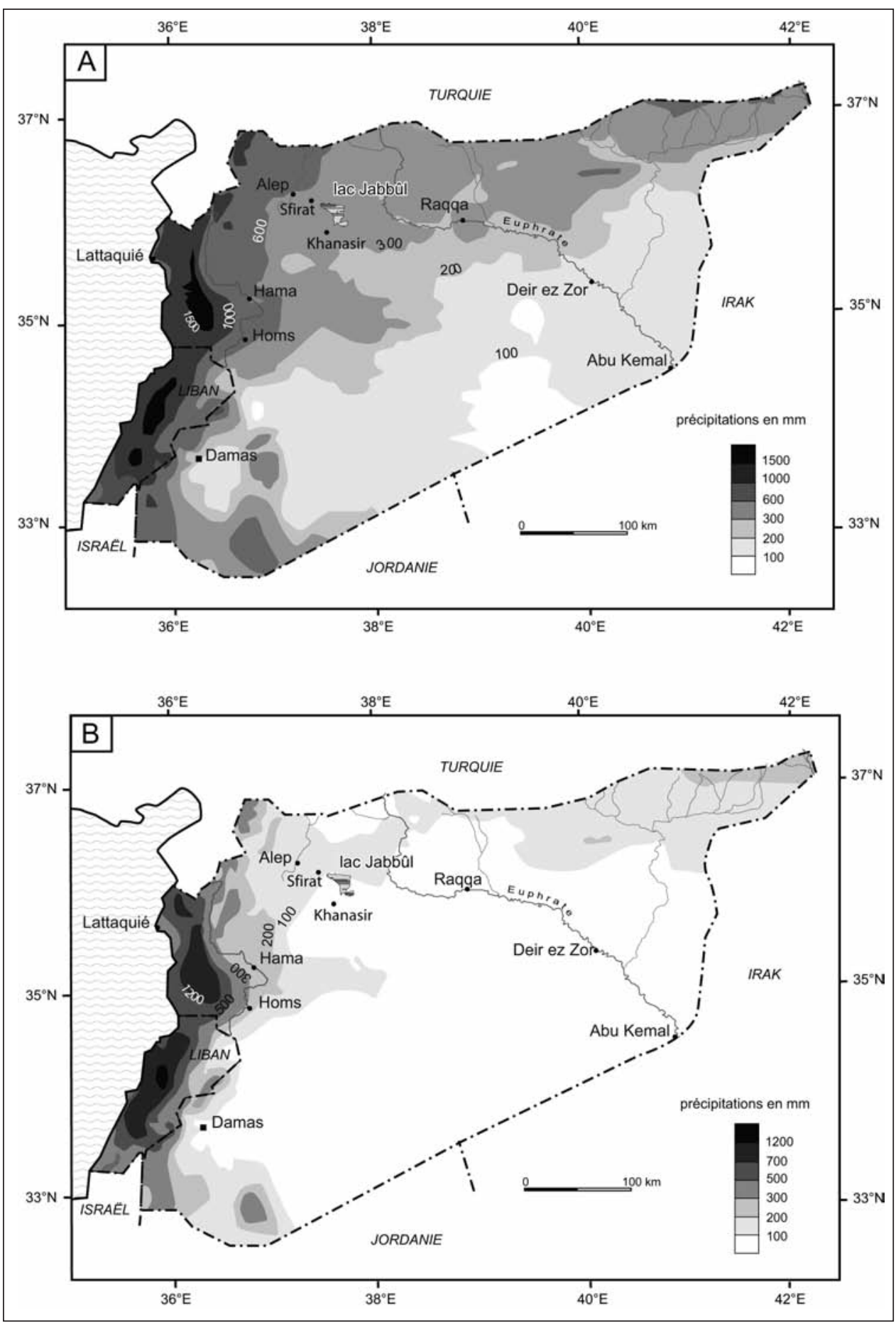

Lithologie, sols, modelé et hydrographie

La région du lac Jabbûl et le plateau d'Alep sont localisés sur une partie mobile du rebord nord de la plaque arabique (Ponikarov et al., 1966 ; Brew et al., 2001). La majeure partie des terrains est façonnée dans des sédiments paléogènes, néogènes et crétacés (Ponikarov et al., 1966 ; Brew et al., 2001) (fig. 4). Ces sédiments sont représentés essentiellement par des calcaires au faciès crayeux ou marno-crayeux peu résistants, à bancs de gypse, avec des intercalations de calcaire silicifié. Des coulées volcaniques du Miocène supérieur, d'origine fissurale (Debelmas et Mascle, 2003) recou- 


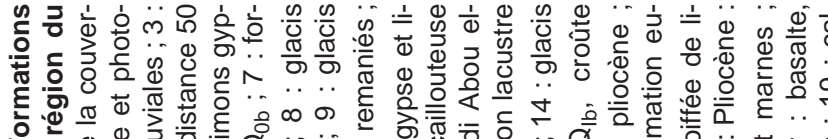

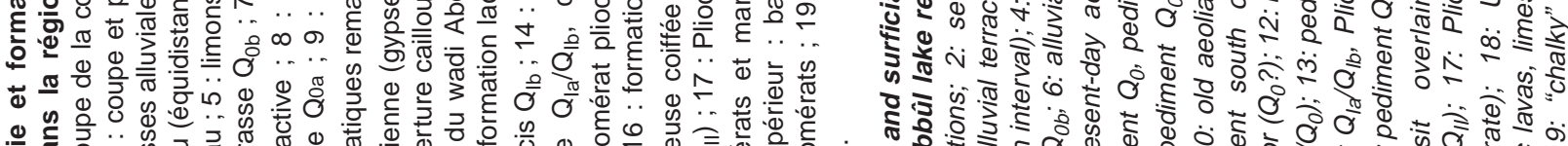

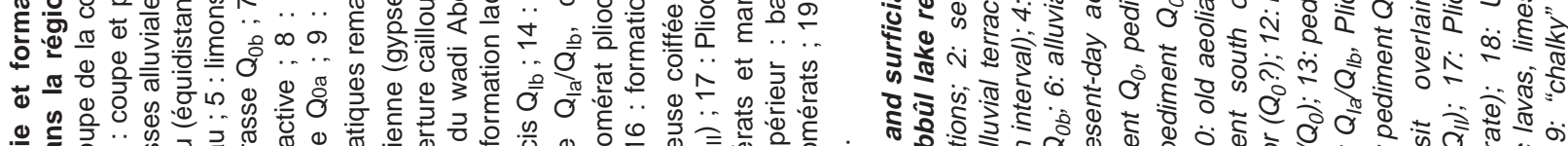

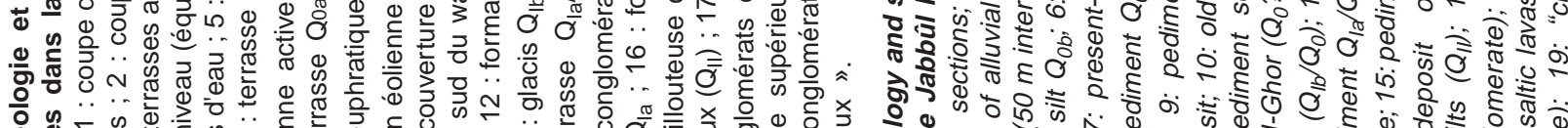

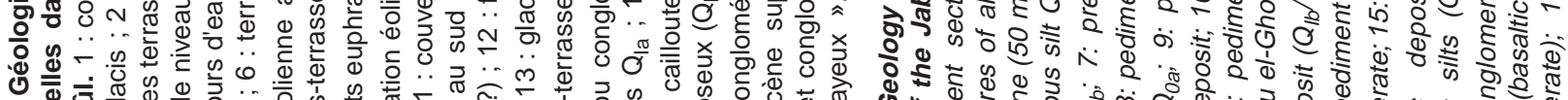

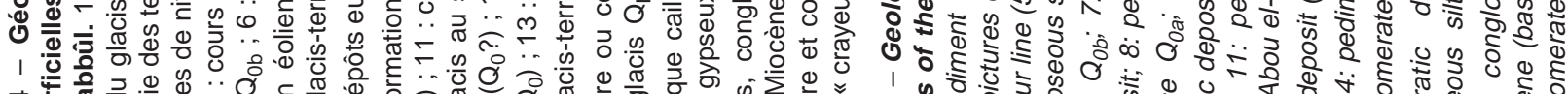

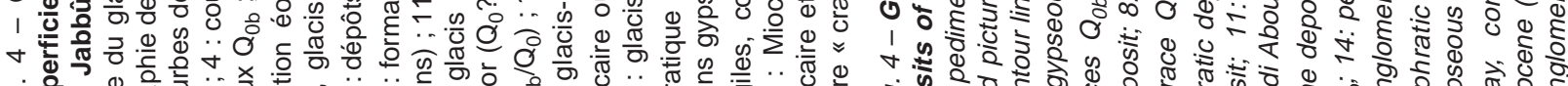
安

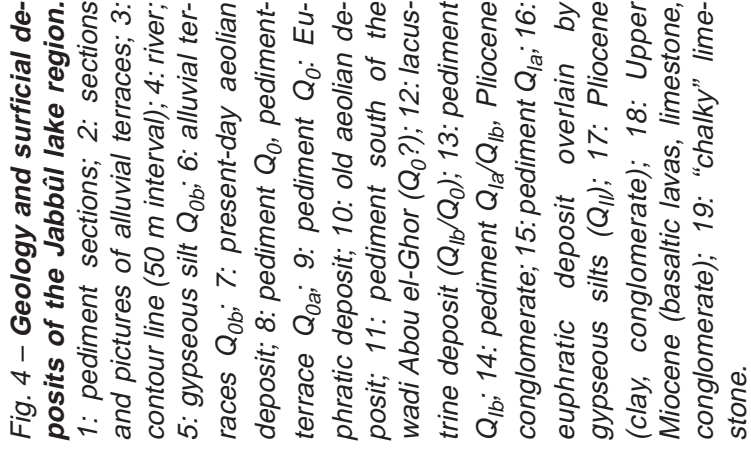
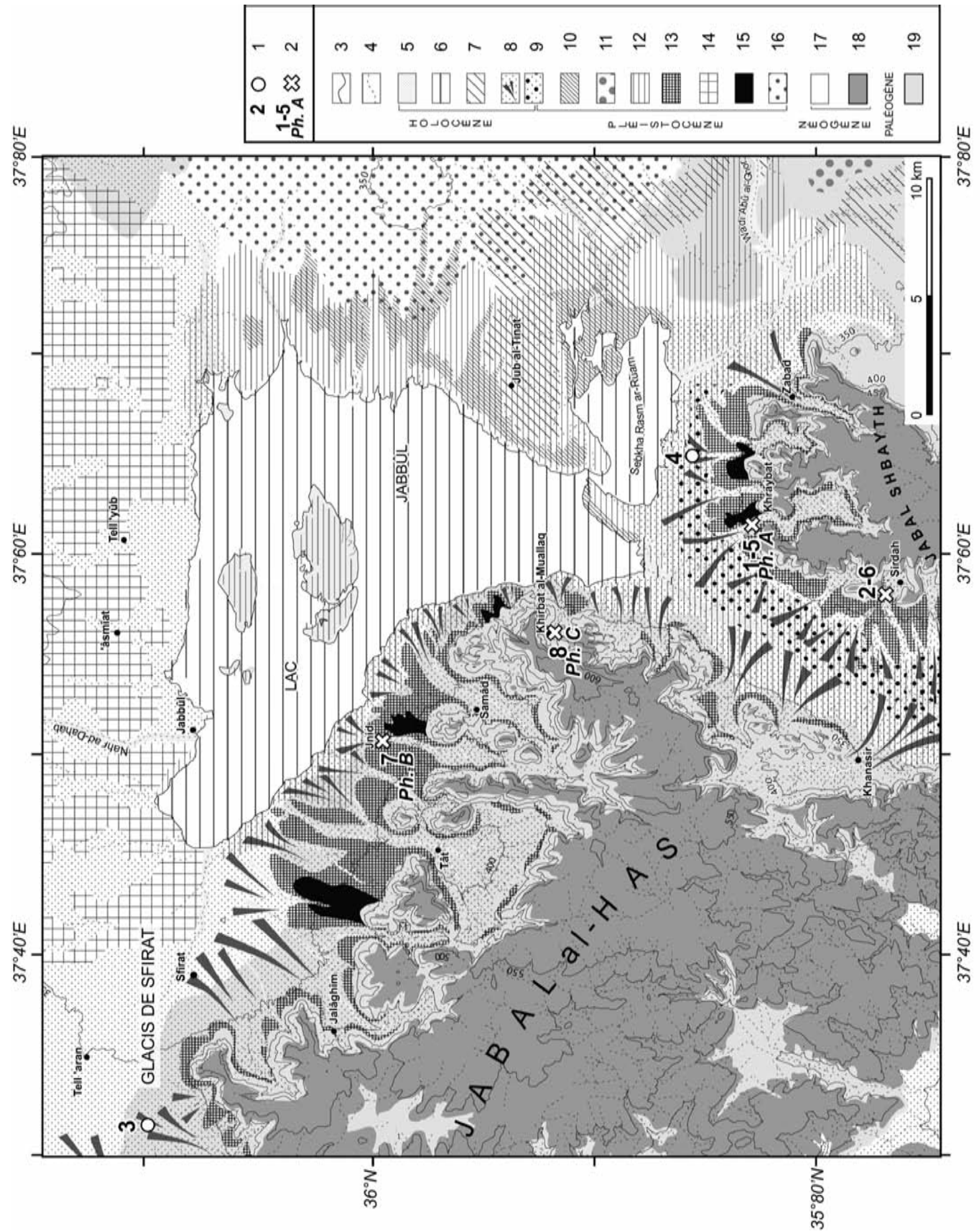
vrent le calcaire des plateaux sur une épaisseur de $10 \mathrm{~m}$ en moyenne (Ponikarov et al., 1966). L'essentiel des dépôts meubles observés aujourd'hui dans la région sont le résultat de l'érosion des basaltes et des calcaires « crayeux ». Cependant, à l'est et au sud se répartissent également des dépôts sablo-caillouteux constitués de roches cristallines arrachées aux montagnes du Taurus et déposées dans la région par un paléocours de l'Euphrate. Les sols de la région dérivent en majeure partie de dépôts meubles très carbonatés : dépôts de versant, couvertures de glacis et alluvions de vallées. Ces sols sont enrichis de particules fines provenant de l'érosion du basalte et de limons éoliens. Ce sont des xérosols, de texture limono-argileuse au nord du lac et dans les vallées des piémonts, à limono-sableuse au sud du lac. Ils sont structurés et granuleux dans les secteurs où la texture est relativement équilibrée, tandis qu'on note une absence de cohésion, témoignant d'une faible pédogenèse, dans les secteurs plus limoneux. Dans tous les cas, la cohésion du sol est fortement limitée par la très faible quantité de matière organique.

Le lac Jabbûl, au centre d'une dépression endoréique, occupe une superficie d'environ $300 \mathrm{~km}^{2}$ en hiver pour une profondeur maximum de $50 \mathrm{~cm}$ et de $2 \mathrm{~m}$ dans la Sebkha Rasm er-Ruam (fig. 4). La partie ouest de la région est occupée par le plateau du Jabal al-Has qui culmine à $650 \mathrm{~m}$, pour une dénivelée d'environ $350 \mathrm{~m}$ par rapport au niveau du lac (niveau de base). Enfin, le Jabal Shbayth, petit plateau allongé vers le sud et occupant la partie sud de la région, culmine à $470 \mathrm{~m}$, avec une dénivellation de $165 \mathrm{~m}$. Les piémonts des plateaux sont constitués d'un glacis principal et de lambeaux de glacis plus anciens (fig. 4) avec une pente de $0,4 \%$ à $3 \%$. Ces formes étagées, recouvertes de croûtes calcaires plus ou moins dures et épaisses, témoignent d'une dissection en plusieurs étapes au Pléistocène, comme le confirme la présence d'outils lithiques pris dans ces croûtes. Elles sont surmontées d'un dépôt meuble hétérométrique à matrice limoneuse dont l'épaisseur varie de $0,50 \mathrm{~m}$ à $5 \mathrm{~m}$ et exceptionnellement $10 \mathrm{~m}$. Les cours d'eau fournissant des informations relatives à la morphodynamique holocène sont localisés dans les Jabals al-Has et Shbayth. Il s'agit d'oueds aux bassins versants de dimension généralement modeste, de 10 à $60 \mathrm{~km}^{2}$.

\section{Les témoignages de l'activité morphogénétique à l'Holocène}

\section{Dépôts alluviaux, morphogenèse et chronologie}

Emboîtées dans les glacis-terrasses du Pléistocène, deux séries de formations holocènes témoignant de deux phases principales de morphogenèse se succèdent (les coupes sont localisés à la fig. 4).

La première est marquée par une nappe alluviale constituée de deux unités distinctes. Dans les vallées des plateaux, cette formation nommée $\mathrm{Q}_{0 \mathrm{a}}$ selon la chronostratigraphie utilisée en Syrie (Besançon et Sanlaville, 1984 ; Geyer et Sanlaville, 1991 ; Besançon, 1994 ; Sanlaville, 1979) est caractérisée par une couche basale hétérométrique à matrice limoneuse contenant des outils proto-néolithiques (entre 10100 et 9600 ans BP), surmontée d'une couverture limoneuse (fig. 5, coupe 1 ; fig. 6A et fig. 7). Sur les piémonts, ces alluvions sont peu évoluées. En revanche, dans une vallée au moins (vallée de Sirdah, à l'ouest du Jabal Shbayth (fig. 4), la formation montre un banc limono-sableux dont la structure granulaire et la bonne aération témoignent d'une pédogenèse (fig. 5, coupe 2). Ce paléosol contient des outils du Néolithique PPNB (Pre Pottery Neolithic B, période culturelle s'étendant de 9600 à 8000 ans BP). Ces deux unités superposées s'observent également en couverture des glacis et des glacis-cônes les plus récents. C'est le cas sur le piémont du Jabal al-Has (fig. 8, coupe 3 et fig. 7), tout comme sur le piémont du Jabal Shbayth, bien que la couverture du glacis diffère quelque peu par la présence d'une unité inférieure sableuse (fig. 7, coupe 4 et fig. 7). La position des terrasses dans le prolongement des glacis-cones indique que ces formes sont contemporaines. Cependant, la couverture des glacis n'est pas datée, si bien que nous les nommons $\mathrm{Q}_{0}$ sans pouvoir préciser. En nous fondant sur les artefacts trouvés dans les coupes (en l'absence de datations isotopiques), on considère que cette nappe alluviale s'est mise en place au minimum lors de la période de transition vers l'Optimum climatique holocène pour l'unité inférieure (autour de 9000 ans BP) puis au cours de l'optimum pour l'unité supérieure (autour de 7 000-8 000 ans BP), période durant laquelle les précipitations sont plus abondantes et plus régulières et la température légèrement plus élevée (Sanlaville, 1996 ; Ayalon et al., 1999 ; Rossignol-Strick, 1999 ; Bar-Matthews et al., 2000).

Dans les vallées, l'unité inférieure hétérométrique témoigne de décharges alluviales brutales (coulées de boues), illustrées par l'absence de tri des alluvions, phénomène caractéristique des milieux arides. Le remaniement des sables d'origine taurique sur le piémont du Jabal Shbayth signale qu'un apport d'eau plus important et plus régulier s'établit assez rapidement. Il s'agit donc d'une phase morphogénétique transitionnelle, qui rompt avec la phase précédente marquée par des conditions plus arides avec une activité éolienne. Cette phase reflèterait la modification climatique du début de l'Holocène vers l'optimum climatique, responsable d'un accroissement de l'érosion des versants encore faiblement protégés par la végétation.

L'unité supérieure limoneuse a été mise en place par des écoulements en nappe. Ces alluvions fines et homogènes traduisent un changement de l'activité morphologique précédente, qui s'explique par le contexte bioclimatique achevant de se modifier. En effet, le climat du début de l'Holocène poursuit sa transition vers l'optimum, phase plus humide et plus chaude, marquée par une plus grande régularité des précipitations. Le décapage des sols se généralise alors sur l'ensemble des versants, ce qui semble indiquer que la couverture végétale n'a pas été assez dense pour empêcher l'ablation. Cependant, l'épaisseur de la nappe de 
limons dans les vallées des mesas et le développement d'un sol dans cette unité supérieure indiquent que cette morphogenèse s'est maintenue.

L'impact de l'optimum climatique holocène sur la morphogenèse a été repéré dans les environs immédiats de la région étudiée. Dans la vallée de l'Euphrate en particulier, Geyer et Besançon (1997) signalent que le fleuve a remblayé la plaine alluviale avec un style en tresses au cours de l'Holocène ancien et que, à partir de 8000 à 7000 ans BP, la dynamique s'est inversée lorsque l'Euphrate a commencé à creuser son lit. Par ailleurs, une terrasse assez similaire à celle que nous avons décrite précédemment, datée du PPNB, s'observe également dans un affluent de l'Euphrate (le Sajour), au nord de la région (Besançon et Sanlaville, 1985). Enfin, l'alluvionnement est bien marqué dans le HautKhabur (Haute Mésopotamie) à cette époque (Courty, 1994).

À la phase morphogénétique suivante correspond l'incision de la formation précédente. Les terrasses étant emboîtées, il n'est pas facile d'évaluer la hauteur de l'incision. Cependant, à partir d'une terrasse étudiée dans le site de Khirbet al-Muallaq (coupe 8, fig. 9), on peut l'évaluer à au moins quatre mètres dans les vallées des plateaux. Vers l'aval, une partie des produits de l'érosion s'observe en surface des glacis. Cette nouvelle dynamique marque la fin de l'optimum climatique holocène mais n'est pas datée avec précision. Une nouvelle phase de dépôt fait suite, illustrée par une nappe alluviale $\left(\mathrm{Q}_{0 \mathrm{~b}}\right)$ emboîtée dans la terrasse $\mathrm{Q}_{0 \mathrm{a}}$. Les coupes, épaisses de $1,2 \mathrm{~m}$ à $4 \mathrm{~m}$, montrent, sur toute leur hauteur, soit des alluvions mal classées et grossières (fig. 5, coupes 5 et 6), soit un dépôt plus fin constitué de sables, de graviers et de petits blocs, en minces strates homogènes (fig. 9 , coupes 7 et 8 ; fig. $6 \mathrm{~B}$ et fig. 7). Des couches de limons dans le corps de ces deux dernières coupes signalent
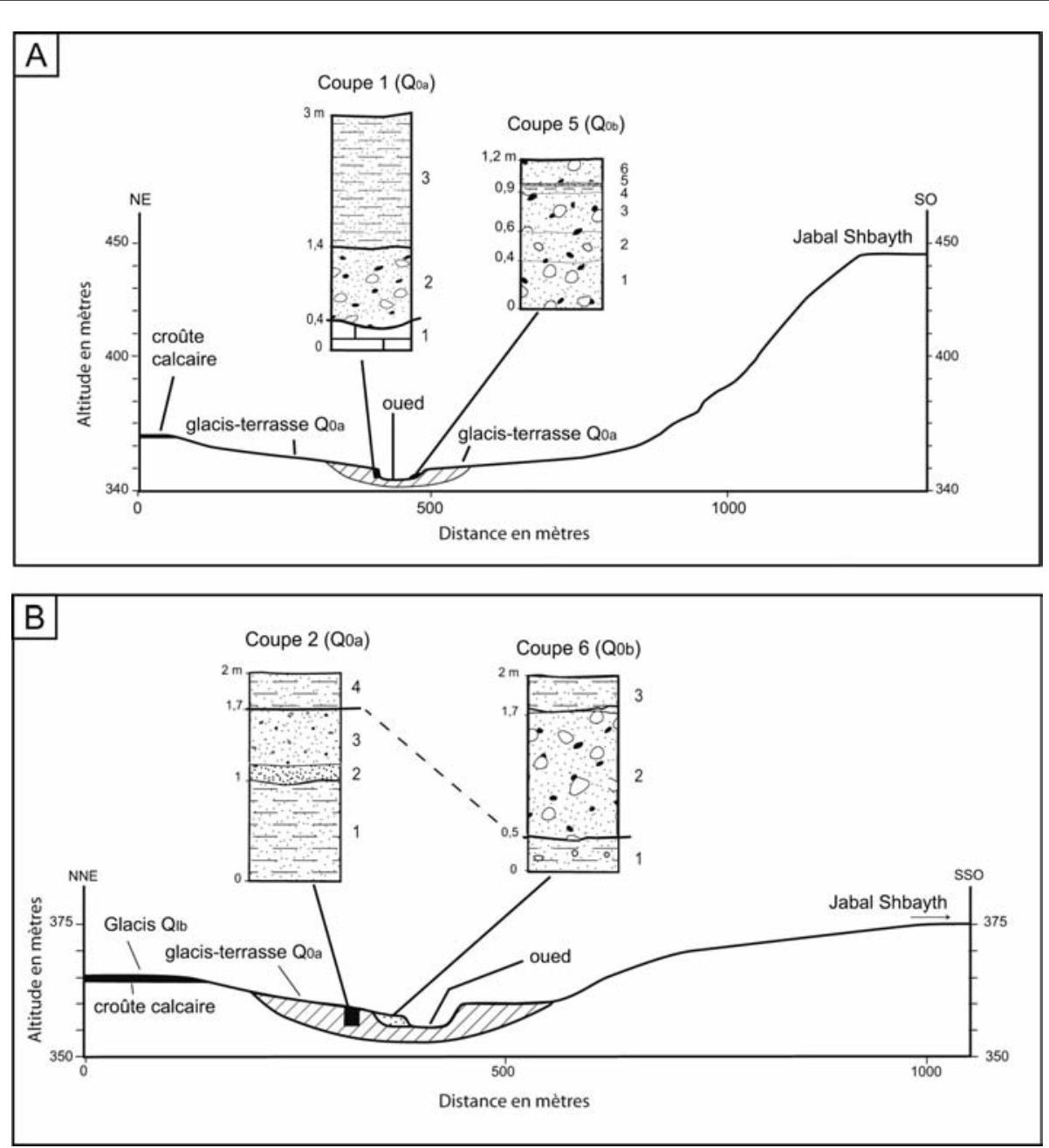

Fig. 5 - Profil transversal des vallées de Khraybat, coupes 1 et 5 et de Sirdah, coupe 2 et 6 (Jabal Shbayth). A : Vallée de Khraybat. Coupe $1-1$ : calcaire éocène raviné ; 2 : couche hétérométrique à matrice limoneuse (non classée) ; 3 : couche limono-sableuse. La couche 2 contient de I'industrie lithique du Néolithique. Coupe $5-1,3,6$ : couches hétérométriques à matrice limonosableuse ; 2 : limons, sables et rares blocs ; 4 : couche limono-sableuse (trace de pédogenèse : micro-organismes, agrégats) ; 5 : couche de sables fins et de limons. Tessons de céramique du Bronze moyen présents dans toute la coupe. B : vallée de Sirdah. Coupe 2 - 1 : couche sablo-limoneuse (apparentée à un paléosol de type xérosol : traces de micro-organismes, agrégats) ; 2 : couche sablo-granuleuse (non classée) ; 3 : couche sablo-limoneuse à cailloux (non classée) ; 4 : sol limono-sableux. Les couches 1,2 et 3 contiennent de l'industrie lithique du Néolithique. Coupe 6 - 1 : identique au niveau « 1 » de la coupe $2 ; 2$ : couche hétérométrique non classée ; 3 : identique au niveau « 4 » de la coupe 2. La couche 2 contient de la céramique de la période romaine.

Fig. 5 - Cross profiles of Khraybat valley, sections 1 and 5, and Sirdah valley section 2 and 6 (Jabal Shbayth). A: Khraybat valley. Section 1 - 1: eroded Eocene limestone; 2: heterometric deposit in a silty matrix; 3: silty-sandy deposit. The layer 2 contains Neolithic artefacts. Section 5 1, 3 and 6: heterometric deposit in a silty-sandy matrix; 2: silt, sand and rare pebbles; 4: silty and sandy layer with pedogenetic traces; 5: layer of fine sands and silts. Middle Bonze Age artifacts are found throughout the whole section. B: Sirdah valley. Section 2 - 1: silty-sandy layer with traces of palaeosol; 2: layer of sand and granules (ungraded); 3; silty-sandy layer with pebbles; 4: silty-sandy soil. Layers 1, 2, 3 contain Neolithic artefacts. Section 6 - 1: silty-sandy layer with traces of palaeosol; 2: ungraded heterometric layer; 3: silty-sandy soil. Layer 2 contains Roman artefacts.

la présence d'au moins deux unités. L'absence de tri des alluvions et l'abondante matrice limono-sableuse signalent une mise en place par des coulées de boue. En parallèle, les nappes de limons témoignent d'épisodes morphologique- 

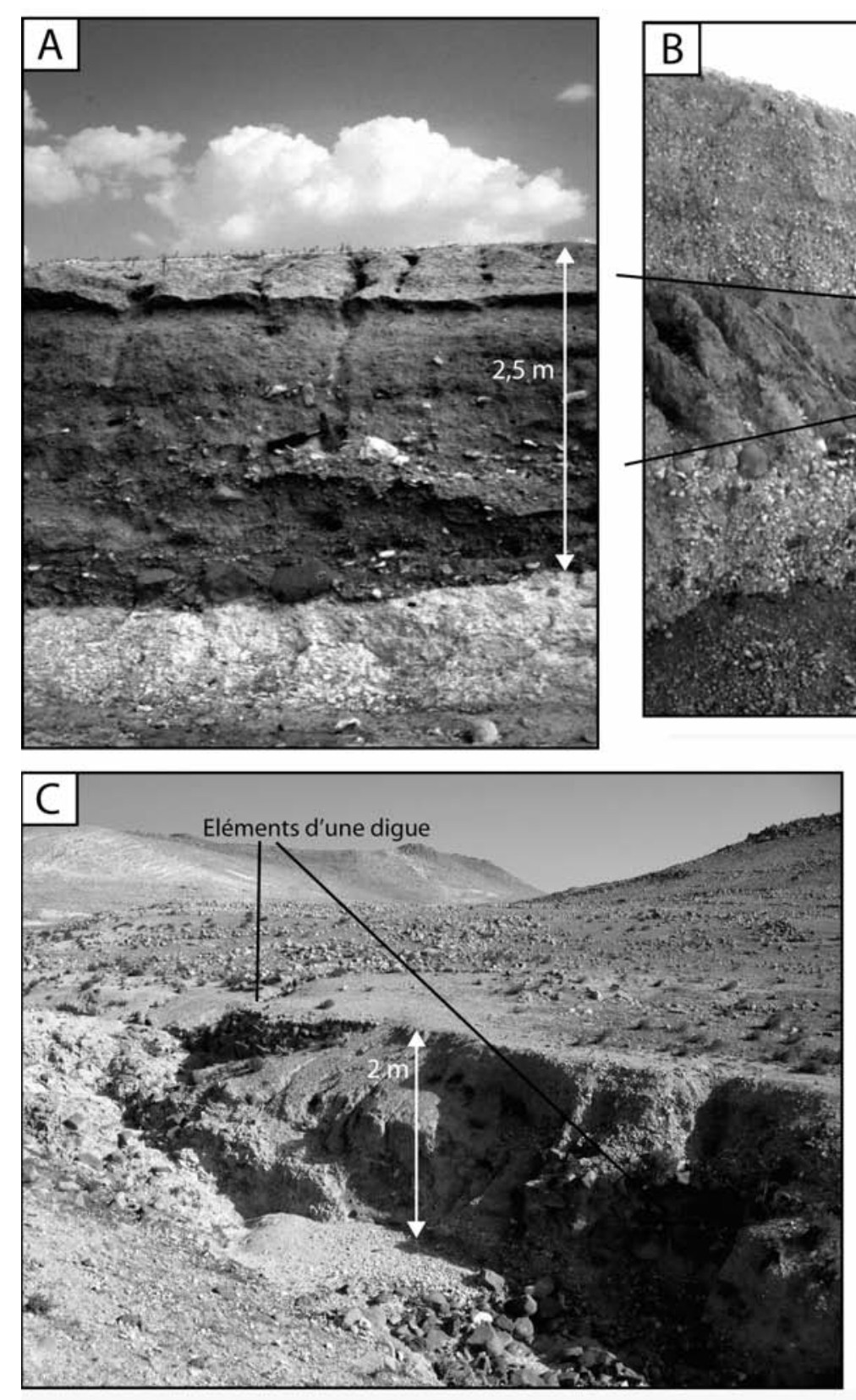

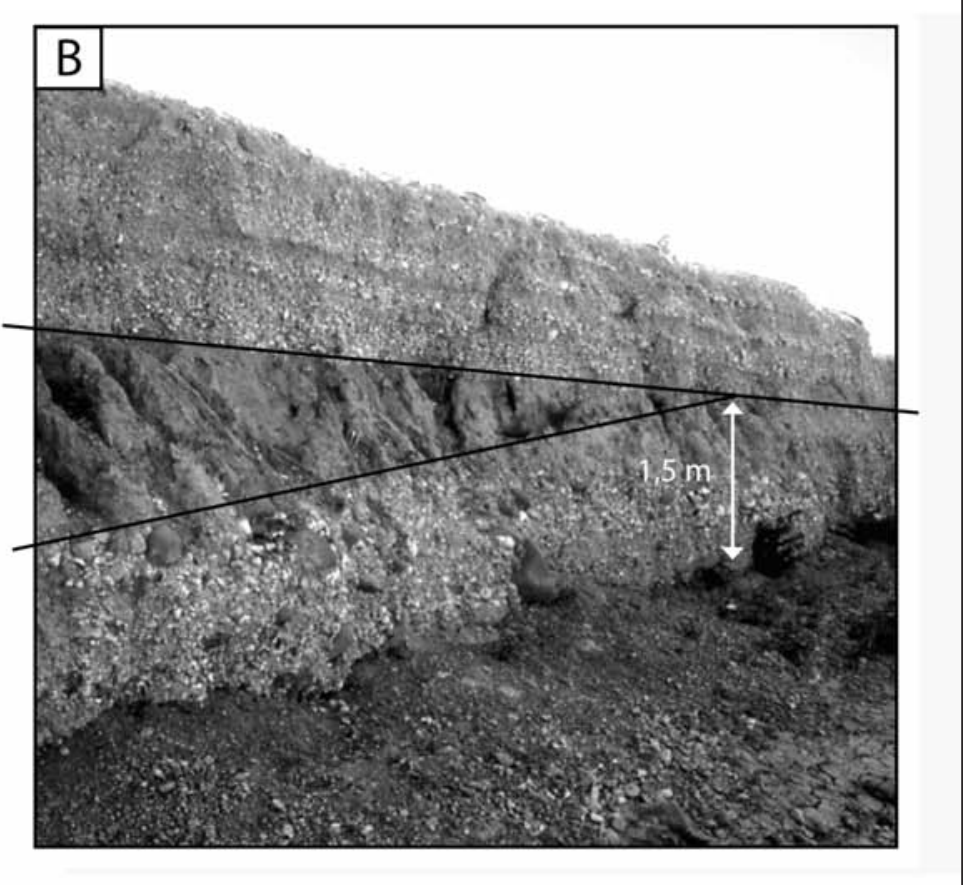

Fig. 6 - Terrasses holocènes dans la région du lac Jabbûl. A : terrasse de l'Holocène ancien (Q0a) sur le calcaire " crayeux » éocène (piémont du Jabal Shbayth, coupe 1) ; B : terrasse postromaine (Q0b) (piémont du Jabal al-Has, coupe 8) ; C : terrasse post-byzantine (Q0b) (Jabal alHas, coupe 7).

Fig. 6 - Holocene terraces in the Jabbûl lake region. A: early Holocene terrace (Q0a) under the "chalky" Eocene limestone (Jabal Shbayth piedmont, section 1); B: post-Roman terrace (QOb) (Jabal al-Has piedmont, section 8); C: post-Byzantine terrace (QOb) (piedmont, section 7).

l'ultime dépôt avant l'incision subactuelle. Par ailleurs, il s'agit du seul dépôt alluvial contenant ce type de céramique mis en évidence dans la région. Il est donc possible que ment plus calmes. De la céramique du Bronze moyen (2 100 à 1600 av. J.-C.) est présente dans une des nappes alluviales (fig. 5, coupe 5). La céramique de l'époque romaine (64 av. J.-C. à $395 \mathrm{AD}$ ) est enterrée dans deux autres dépôts alluviaux étudiés (fig. 5, coupe 6 et fig. 9, coupe 7). Enfin, de la céramique de l'époque byzantine (395 à $636 \mathrm{AD}$ ) a été relevée dans une coupe (fig. 9, coupe 8). Ces restes d'âges variés compliquent l'interprétation de la succession des événements. Il s'agit soit de plusieurs phases d'alluvionnement, au cours de périodes différentes mais rapprochées (en ce qui concerne les périodes romaine et byzantine, fig. 7), soit d'une unique phase de morphogenèse, des restes de céramique d'âges différents ayant pu être associés à un même épisode d'alluvionnement.

Un doute subsiste quant à la nappe alluviale datée du Bronze par la céramique. Située à l'aval d'un site du Bronze, sa position dans le paysage indique qu'il s'agit de sa mise en place soit en réalité contemporaine d'un épisode morphogénétique bien postérieur à l'époque du Bronze, peut-être post-romain, sinon post-byzantin. Les nappes alluviales datées des époques romaine et byzantine par la céramique ne se trouvent pas dans toutes les vallées. Pourtant, il existe des sites byzantins en amont d'une vallée (vallée de Sirdah) dans laquelle existe un dépôt « romain ». La question de la succession de ces nappes alluviales dans le temps et dans l'espace reste donc posée. En particulier, nous ne savons rien de l'éventuelle phase d'érosion ayant suivi le dépôt des alluvions « romaines » et précédé celui des alluvions «byzantines », si tant est que ces deux phases soient distinctes. Mais, au-delà de ces questions de chronologie, le fait essentiel est l'absence de témoignage d'un décapage des sols dans les alluvions romaines et byzantines. En particulier, il n'existe pas d'épaisse couche de limons dans ces nappes alluviales. 


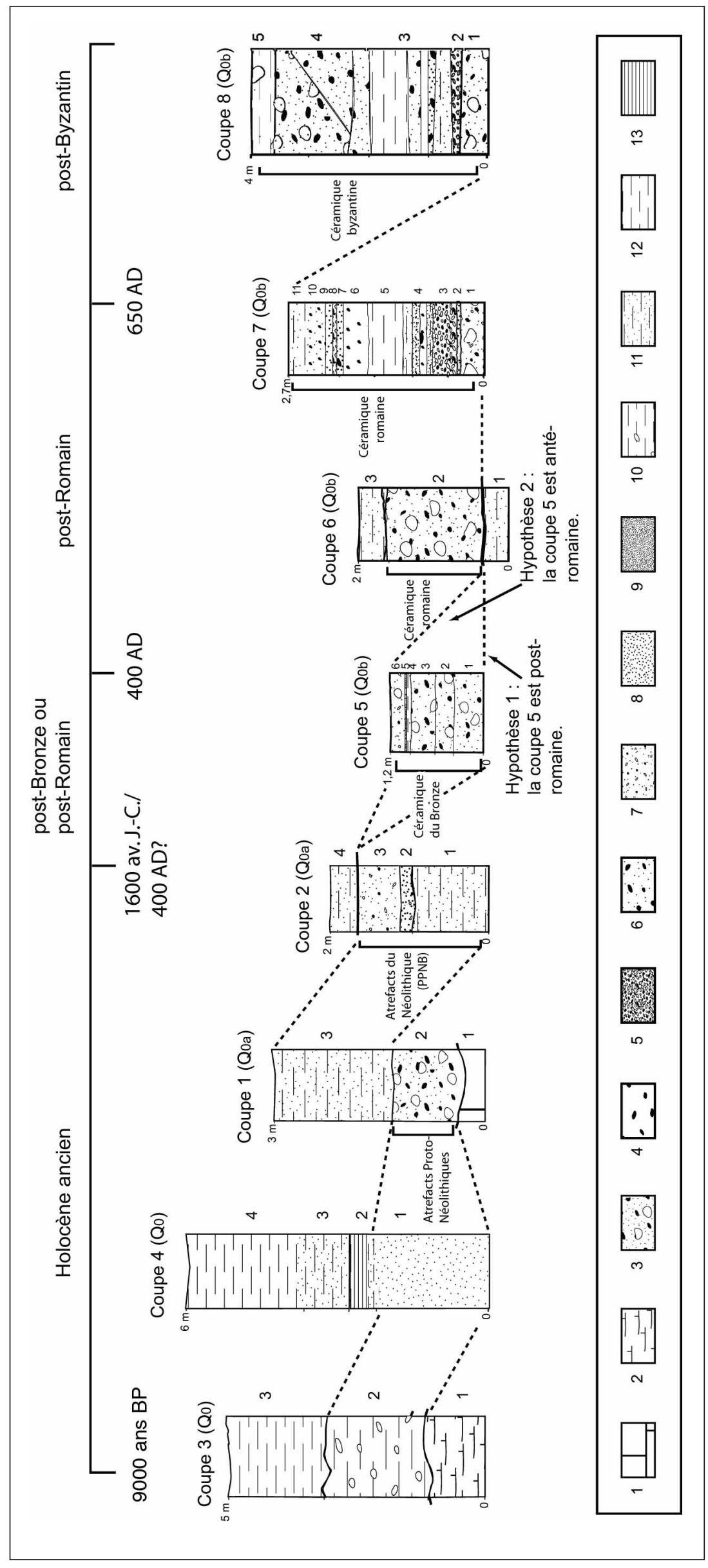

Fig. 7 - Chronologie relative des formations holocènes. 1 : calcaire éocène ; 2 : calcaire éocène altéré ; 3 : dépôt hétérométrique ; 4 : cailloux ; 5 : graviers ; 6 : sables et cailloux ; 7 : sables et graviers; 8 : sables grossiers ; 9 : sables fins ; 10 : limons et blocs ; 11 : limons sableux ; 12 : limons ; 13 : argiles.

Fig. 7 - Relative chronology of Holocene formations.1: Eocene limestone; 2: weathered Eocene limestone; 3: heterometric deposit; 4: pebbles; 5: gravels; 6: sand and pebbles; 7: sand and gravel; 8: coarse sand; 9: fine sand; 10: silt and cobbles; 11: silt and sand; 12: silt; 13: clay.

Ces différents épisodes morphogénétiques ont été mis en évidence à proximité immédiate de la région étudiée. Ainsi, dans la vallée de l'Euphrate, il existe une terrasse du Bronze et une série de « dépôts complexes postérieurs » (Geyer et Besançon, 1997). Dans le bassin versant du Sajour, Besançon et Sanlaville (1985) ont relevé des terrasses grossières contenant de la céramique du Bronze dans une vallée et de la céramique romaine dans une autre. Enfin, dans le haut Balikh, un alluvionnement « post-romain »a été noté par Wilkinson (1999). Ces informations montrent que, à partir de l'époque romaine au moins, l'activité morphogénétique reprend dans la région. Cette activité, visiblement modeste, pourrait être associée à un léger changement climatique, illustré par des précipitations mieux réparties dans l'année. Pour l'instant, aucune preuve paléoécologique ne nous permet de l'affirmer pour la région. Mais des témoignages du retour à un climat plus humide ont été relevés ailleurs au Proche-Orient et pourraient étayer nos observations. C'est le cas dans la région de Palmyre en Syrie où des limons organiques ont été datés de $1860 \pm 70$ ans BP et de $1930 \pm 30$ ans BP (Besançon et al., 1997), en Haute Jézireh où on note le développement d'un sol et l'augmentation du pollen d'arbres à la même période (Rösner et Schäbitz, 1991), dans la vallée du Balikh en Turquie (Rosen, 1997), dans le Levant sud, où la remontée du niveau de la mer morte indique une période plus arrosée qui atteindrait son paroxysme vers $90 \mathrm{AD}$ (Enzel et al., 2003 ; Bruins, 1994 ; Yakir et al., 1994 ; Issar et al., 1991) ou encore sur la côte israélienne un peu plus tardivement, où l'analyse d'une carotte marine indique l'existence d'une « période humide » entre 1700 et 1000 ans BP (Schilman et al., 2001) (fig. 10). 
Cet épisode d'alluvionnement de l'époque romaine est suivi par une ou plusieurs phases d'incision. Cette incision a été si importante (jusqu'à $4 \mathrm{~m}$ pour la terrasse byzantine) qu'elle témoigne apparemment d'une dynamique des cours d'eau non négligeable. Pourtant, un carottage réalisé dans le lac Jabbûl n'a montré, côté est, qu'une accumulation de cinq mètres de limons ; au centre du lac, un autre carottage plus ancien indique une épaisseur de dix mètres de sédiments. Or, nous savons que cette sédimentation s'est amorcée dès avant l'Holocène, car les formations éoliennes édifiées à la suite de l'ultime creusement du lac sont surmontés de silex du Kébarien (16 500 ans BP). L'accumulation sédimentaire dans le lac Jabbûl est donc très faible depuis la fin du dernier glaciaire. Cela a plusieurs significations : d'une part, l'alluvionnement historique a été réduit et limité à un chenal étroit (une dizaine de mètres au maximum), d'autre part, l'érosion des nappes alluviales historiques a fourni peu de débris ; enfin, cette érosion est restée modeste. La question de l'origine de l'incision reste posée. La fraîcheur des coupes milite en faveur d'un événement récent qui pourrait être le petit âge glaciaire, comme le suggèrent les conclusions d'E. Fouache (1999) en ce qui concerne la terrasse d'Olympie.

\section{L'alluvionnement majeur de l'époque romaine n'est pas systématique en Syrie}

L'étude de la région du lac Jabbûl révèle une dynamique morphogénétique limitée durant la période située entre la fin de l'optimum climatique et l'époque contemporaine. Toutes les terrasses qui se rapportent à cette période, hormis une d'entre elles (qui contient de la céramique du Bronze moyen), sont au minimum post-romaines d'après la céramique (fig. 8 et fig. 10). Si les dépôts sont parfois épais (jusqu'à $4 \mathrm{~m}$ : fig. 9, coupe 8), ils comblent des chenaux si étroits que l'on ne peut les associer à un phénomène d'érosion généralisée de la couverture pédologique à l'échelle régionale. La terrasse byzantine de Khirbat al-Muallaq (fig. 9, coupe 8) est, à cet égard, révélatrice : son étude détaillée a révélé qu'elle était circonscrite presque au seul site d'occupation byzantin. Vers l'aval, l'incision du cours d'eau diminue rapidement ainsi que l'épaisseur de la nappe alluviale, emboîtée dans les sédiments holocènes anciens. Son épais- seur est importante au sein du site archéologique, car l'incision qu'elle comble est profonde (4 m). Cependant, des témoignages d'endiguement révèlent que l'incision existait déjà au moment de l'installation byzantine (fig. 6C), ce que confirme également la présence des habitations en bordure immédiate de l'oued. Cette situation est encore plus remarquable au sud de la région du lac Jabbûl, dans un contexte naturel similaire (Geyer, communication orale). Dans ce secteur, il n'y aurait qu'une seule nappe alluviale holocène. L'absence de tessons et la présence fréquente en position sommitale d'artefacts néolithiques suggèrent, avec toute la prudence nécessaire, qu'elle date du début de l'Holocène (donc de la phase d'optimum climatique). Ensuite, le secteur paraît marqué par une surprenante stabilité morphogénétique.
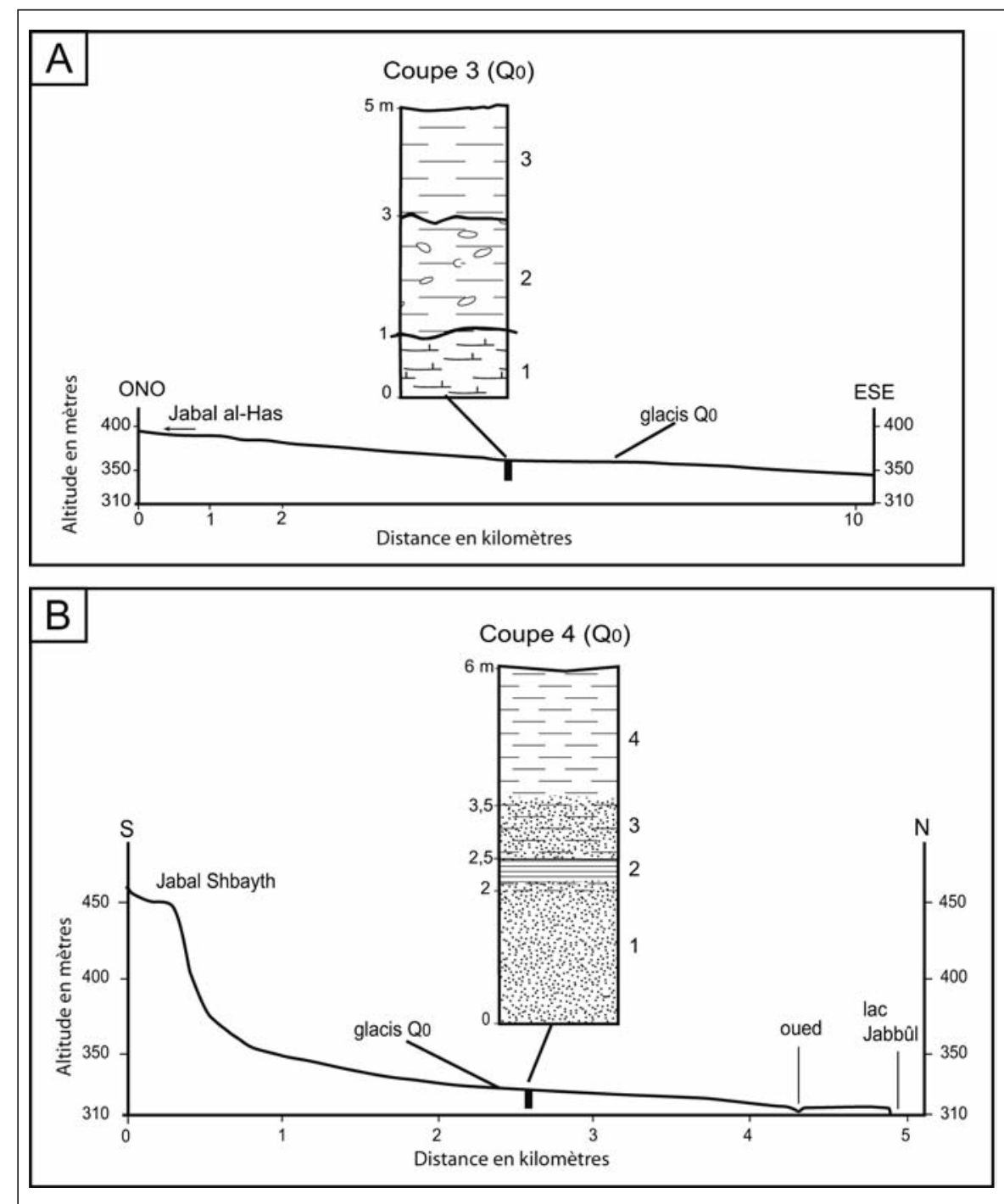

Fig. 8 - Profils topographiques de deux glacis et coupes 3 et 4 . A : Glacis de Sfirat (NO du lac Jabbûl). Coupe $3-1$ : calcaire éocène altéré ; 2 : blocs dans une matrice limoneuse carbonatée ; 3 : limons et argiles. B : glacis Nord du Jabal Shbayth (S du lac Jabbûl). Coupe 4 - 1 : sables gris ; 2 : couche argilo-sableuse ; 3 : limons avec graviers ; 4 : limons rougeâtres.

Fig. 8 - Topographic profiles of two pediments and sections 3 and 4. A: Sfirat pediment (NW of the Jabbûl Lake). Section 3 - 1: weathered Eocene limestone; 2: pebbles in a calcareous matrix; 3: silt and clay. B: North Jabal Shbayth pediment (S of Jabbûl lake). Section 4 - 1: grey sand; 2: layer of clay and sand; 3: silt and granules; 4: reddish silt. 

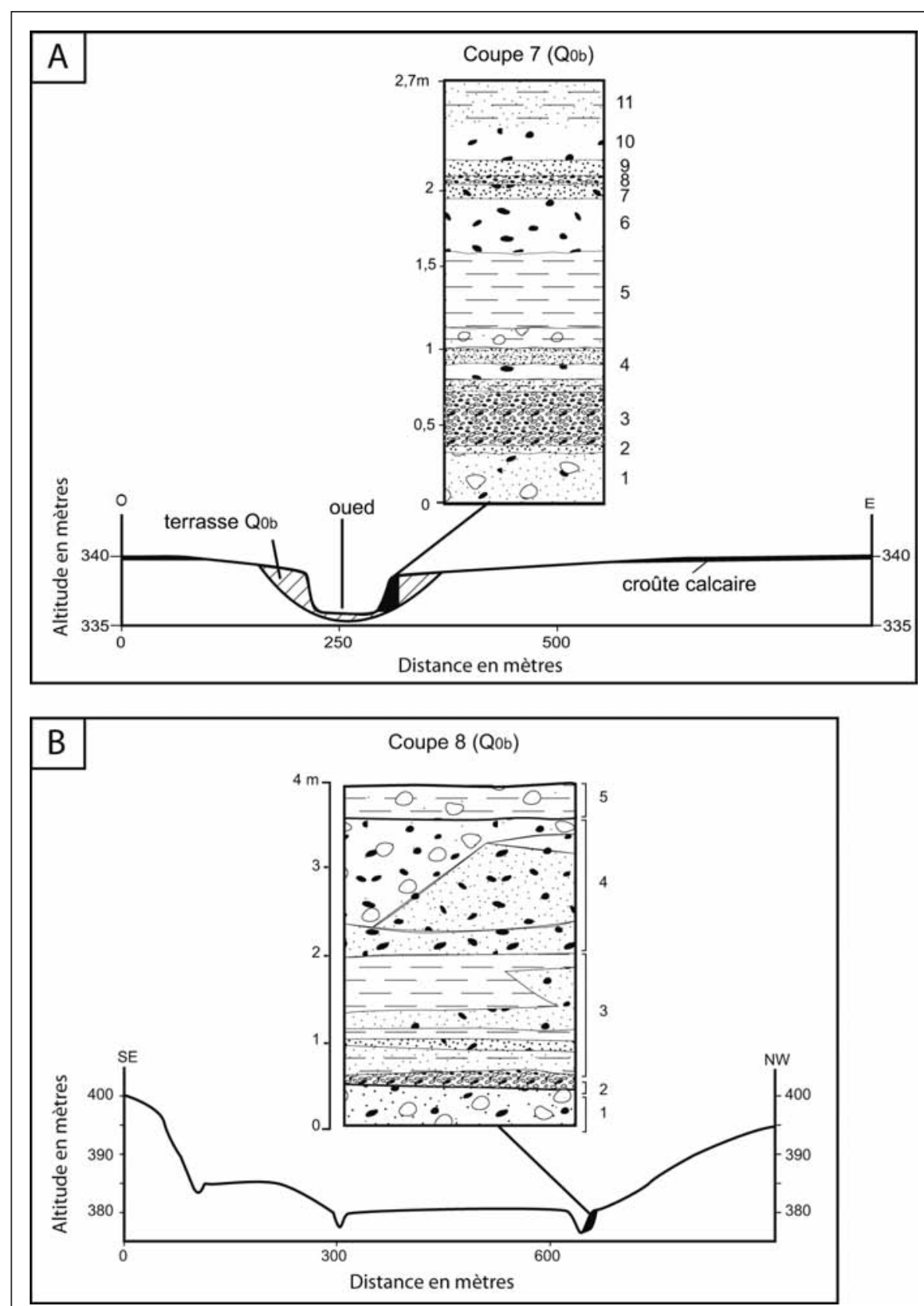

Au vu du faciès des nappes alluviales, on peut penser que des phénomènes climatiques courts et brutaux, associés à une intense mise en valeur agricole à proximité immédiate des installations humaines pourraient être à l'origine de l'édification des quelques terrasses historiques observées dans la région du lac Jabbûl. La coupe citée précédemment (fig. 9, coupe 8) en est une bonne illustration : l'absence de trace de pédogenèse dans les dépôts, la fraîcheur des tessons retrouvés et leur présence dans toute la hauteur de la coupe suggèrent une mise en place rapide, en quelques décennies et sans transport notable. Mais rien n'atteste une reprise majeure et généralisée de l'érosion liée à un événement climatique ou anthropique dans la région.

Ces observations sont en contradiction avec de nombreuses études menées ailleurs au Proche-Orient et en Méditerranée, qui décrivent d'épaisses nappes alluviales et
Fig. 9 - Coupes 7 et 8 , terrasses alluviales, piémont du Jabal al-Has. A : profil du glacis à Jnid (Jabal al-Has). Coupe $7-1$ : couche hétérométrique à matrice limono-sableuse ; 2 : sables grossiers ; 3 : graviers ; 4 : alternance de couches sablo-limoneuses et sablocaillouteuses ; 5 : couche limoneuse ; 6 : cailloux ; 7 : couche sablo-caillouteuse ; 8 : graviers ; 9 : sables grossiers ; 10 : couche caillouteuse à faible matrice limoneuse ; 11 : limons et sables. La céramique de l'époque romaine est présente dans toute la coupe. $B$ : profil de la vallée à Khirbat al-Muallaq (Jabal al-Has). Coupe $8-1$ : couche hétérométrique à lentilles sableuses ; 2 : couche caillouteuse ; 3 : limons avec lentilles sablocaillouteuses ; 4 : couche sablo-caillouteuse à caillouteuse ; 5 : sol limoneux (xérosol). La céramique de l'époque byzantine est présente dans toute la coupe.

Fig. 9 - Sections 7 and 8, alluvial terraces, Jabal al-Has piedmont. A: Jabal al-Has pediment profile at Jnid (Jabal alHas). Section $7-1$ : coarse deposit in a sandy matrix; 2: coarse sand; 3: gravels; 4: alternation of sandy-silty beds and sand and granules beds type; 5: silty layer; 6: pebble deposit; 7: layer of sand and gravels; 8: gravels; 9: coarse sand; 10: pebble layer with silty matrix; 11: silt and sand. Roman artifacts are found throughout the whole section. B: valley profile at Khirbat al-Muallaq (Jabal alHas). Section 8 - 1: heterometric layer with sand lenses; 2: layer of gravels; 3 : silt with sand and gravel lenses; 4: layer with sand and pebbles; 5: silty soil (xerosol). Byzantine artifacts are found throughout the whole section.

colluviales, la plupart interprétées comme étant le résultat d'une intense érosion des sols au cours des périodes hellénistique et romaine, voire jusqu'au haut Moyen Âge.

Une longue bibliographie témoigne de la récurrence de ce phénomène. Sans entrer dans les détails, on peut signaler les synthèses déjà anciennes mais riches de Vita-Finzi (1969) et de Dufaure (1984) et, plus récemment, de Fouache (1999). Signalons également les travaux menés en Italie, au Maghreb ou encore en Bythinie, où des terrasses hellénistiques et romaines ont été mises en évidence (Neboit, 1984, 1991 ; Ballais, 1984, 1995 ; Geyer et Lefort, 2003). Enfin, on peut également citer les travaux menés en France, en Turquie, en Grèce, à Chypre ainsi qu'en Syrie où une reprise de l'érosion torrentielle est relevée entre la fin de la période antique et le début du Haut Moyen Âge (ou à la fin de la période byzantine en Méditerranée Orientale) (Ballais et al., 1993, Ballais et Crambes, 1993 ; Wilkinson, 1999 ; Fouache, 1999 ; Devillers, 2003 ; Geyer et Sanlaville 1991 ; Besançon et Sanlaville, 1985 ; Sanlaville, 1979). 


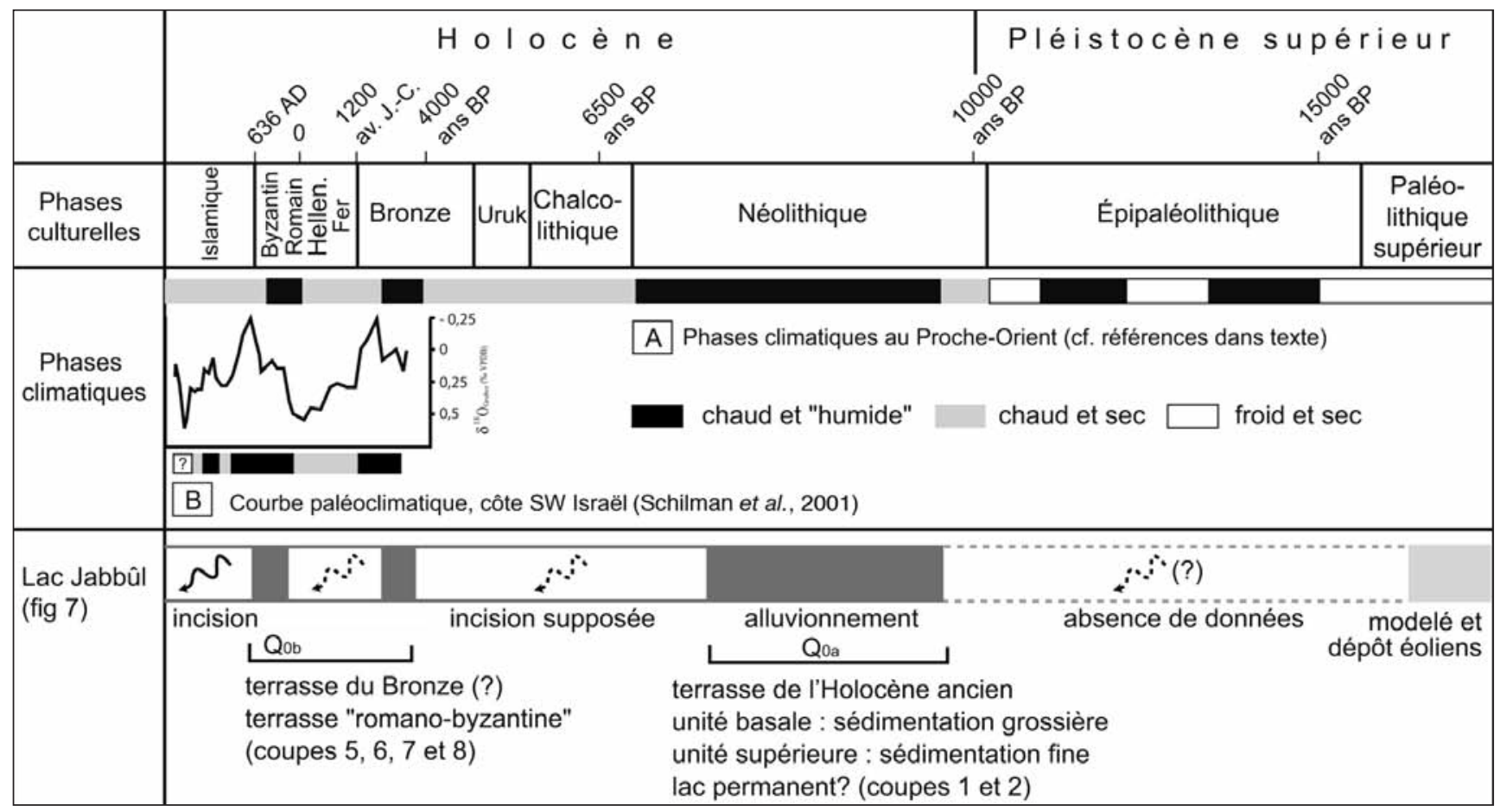

Fig. 10 - Chronologie et morphogenèse durant la fin du Pléistocène et l'Holocène dans la région du lac Jabbûl.

Fig. 10 - Late Pleistocene and Holocene landscape evolution chronology in the Jabbûl lake region.

Pour certains auteurs, l'origine de ces nappes alluviales est à rechercher dans la forte pression humaine entraînant la colonisation d'espaces fragiles, des défrichements et provoquant une rupture d'équilibre en amont des bassins versants (Brückner, 1990 ; Barker, 1995 ; Fouache, 1999). Ce phénomène est généralement associé à l'action du climat (Ballais, 1995 ; Ballais et al., 1993 ; Neboit, 1984, 1991 ; Neboit-Guilhot, 2003 ; Wilkinson, 1999), ne serait-ce qu'en raison de sa fréquence dans le bassin méditerranéen aux mêmes dates. Pour d'autres, la déprise agricole aurait été la cause prédominante de cet alluvionnement (Dufaure, 1976 ; Besançon et Sanlaville, 1985). Mises en place dans des milieux parfois différents, dans des contextes socio-économiques variés, toutes ces nappes alluviales n'ont pas la même origine. En revanche, elles sont toujours épaisses, contiennent presque systématiquement une importante fraction limono-argileuse et témoignent, en cela, d'une phase d'intense érosion des sols arables.

La première explication que l'on peut donner à la faible activité morphologique observée dans la région du lac Jabbûl à partir de l'Holocène moyen est d'ordre naturel, en raison de sa situation et de la lithologie. Cette région s'inscrit en effet dans les marges arides et, surtout, elle est protégée des influences maritimes par une barrière montagneuse. Il est vrai que les régions de marge enregistrent rapidement toutes modifications du climat, même minimes, et que ces modifications ont des répercussions sur l'évolution morphogénétique. Mais, dans le cas des steppes du nord de la Syrie, l'orographie côtière, en bloquant les dépressions méditerranéennes, a empêché que s'amorce un retour généralisé de l'érosion des versants et des sols suivi d'un comblement des chenaux tel qu'il s'est manifesté dans le reste du bassin méditerranéen. Un phénomène identique a eu lieu avec le Taurus qui a bloqué les dépressions en provenance du nord. Par ailleurs, les plateaux étant coiffés de basalte, on peut supposer, en particulier dans le secteur du jabal Shbayth, que la fourniture de matériau par recul du versant a été réduite.

Cependant, ce début d'explication n'est pas entièrement satisfaisant car l'effet d'abri ne semble pas avoir fonctionné aussi efficacement au début de l'Holocène. Il faut donc comprendre que l'optimum climatique de l'époque romaine (s'il a existé) reste modeste et peu comparable à celui du début de l'Holocène. Il n'est bien sûr ni possible ni souhaitable de se limiter à des explications d'ordre naturel, dans un secteur si fortement mis en valeur à l'époque romanobyzantine ; il est évident que l'Homme a joué un rôle majeur dans l'évolution du paysage. En effet, dans le cas du lac Jabbûl comme dans le reste de la steppe de Syrie du Nord, les prospections archéologiques montrent que l'occupation ne cesse de croître à partir de l'époque hellénistique, pour atteindre un maximum à la période byzantine. Il n'y a pas de départ des populations à l'époque romaine, en particulier pas de départ massif d'agriculteurs, donc pas de déprise. Par ailleurs, si l'on constate un début de migration des sédentaires et une présence plus importante des nomades à l'époque byzantine, le début de la période islamique est encore marqué par une présence humaine non négligeable. L'absence de déprise généralisée est donc un élément d'explication supplémentaire de la faible activité morphologique observée dans la région. 
Qu'en est-il, en revanche, de la pression humaine et de la surexploitation agricole ? Celle-ci est capable de provoquer une érosion des sols, en particulier sur les versants non protégés. Il est certain que la région connaît une forte occupation, mais nous n'avons pas d'élément attestant une éventuelle surexploitation du sol. En revanche, on constate, sur les versants des mesas bordant le lac Jabbûl, une forte concentration de terrasses de culture datant des époques romaine et byzantine. Ainsi, si elle a été intense, l'exploitation du sol s'est opérée en tenant compte des contraintes du milieu naturel et avec un soin extrême, comme en témoigne la très bonne conservation des terrasses. On peut supposer que, sans ces aménagements, l'érosion des versants aurait été plus vive, à l'instar de ce qui s'est passé au début de l'Holocène. D'ailleurs, le décapage généralisé des sols sur les versants à l'Holocène inférieur explique également la faible proportion des limons dans les alluvions historiques.

L'alluvionnement historique limité dans les steppes arides du nord de la Syrie s'explique donc par une série de facteurs dans lesquels les sociétés et les processus naturels sont partie prenante. D'une part, l'épisode climatique de l'Âge classique a eu des répercussions trop faibles dans les steppes $\mathrm{du}$ nord de Syrie pour provoquer, à lui seul, une reprise notable de l'érosion, dans un contexte lithologique où une roche dure prédomine à l'amont des bassins versant. D'autre part, l'impact de l'action humaine sur le milieu naturel a été plutôt positif, dans le secteur du lac Jabbûl au moins. Enfin, le décapage des sols au début de l'Holocène est aussi responsable de la faible fourniture de sédiments fins aux cours d'eau durant les périodes historiques.

\section{Conclusion}

Il ressort de cette étude que l'évolution morphologique de la région à l'Holocène est marquée par deux phases bien distinctes. D'une part, à l'Holocène ancien et moyen, l'activité morphologique est intense, tant sur le plan de l'érosion que sur celui du façonnement des formes de terrasses et de glacis. Cette morphogenèse est à mettre en relation, entre autres, avec l'optimum climatique holocène. D'autre part, l'Holocène récent est marqué par une relative stabilité morphologique. La première phase est bien connue en domaine méditerranéen et aride. En revanche, la seconde est beaucoup plus originale. En effet, contrairement à ce qui se passe dans le bassin méditerranéen, où d'épaisses accumulations limoneuses dans les chenaux illustrent un décapage des sols dès la période hellénistique, les dépôts, bien que parfois épais, ne reflètent aucune érosion généralisée des sols. Cette différence fondamentale n'est pas à imputer à l'immobilisme des composants du milieu dans la région, puisque des nappes alluviales datant du Bronze (?) et des périodes romaine et byzantine ont été observées (fig. 7 et fig. 10), mais plutôt à leur stabilité à partir de la fin de l'optimum climatique holocène. Tout se passe comme si la dynamique morphogénétique avait été fortement inhibée à partir de 6000 ans BP (fig. 10). Le climat et, en parallèle, la configuration orographique du nord de la Syrie, ainsi que les conditions morphostructurales locales, ont joué un rôle majeur dans ce ralentissement des processus. Cette observation apporte un éclairage nouveau sur une région souvent trop hâtivement mise en parallèle avec le bassin méditerranéen tout proche.

Un constat original s'impose donc : malgré leur proximité avec la mer Méditerranée et bien que bénéficiant d'un climat en partie semblable au type méditerranéen, les régions de marges arides du nord de la Syrie ne peuvent être assimilées au reste du bassin méditerranéen pour ce qui relève de l'érosion des versants et des sols. Ici, c'est l'aridité qui est la principale caractéristique du climat et, comme elle perdure depuis la fin de l'optimum climatique holocène, elle est un des principaux freins à l'activité morphologique. Sur le pourtour méditerranéen, d'autres éléments inconnus dans la steppe entrent en jeu. La disposition du relief (pentes vigoureuses, dénivelées importantes) et les précipitations (deux à trois fois plus importantes que dans les marges arides), induisent des phénomènes érosifs d'une tout autre ampleur. À cela s'ajoute la dimension humaine : la forte concentration de l'occupation et la surexploitation des terres en amont des bassins versants notamment, qui débute à l'époque hellénistique, ont favorisé le décapage des sols. Dans la steppe, à l'inverse, malgré une occupation dense à partir de l'époque romaine, les aménagements agricoles ont contribué à freiner l'érosion des versants dans un contexte régional aride.

Ces conclusions, en contradiction avec de nombreuses observations réalisées dans le bassin méditerranéen et au Proche-Orient, participent d'un renouvellement nécessaire de nos connaissances et de notre manière d'appréhender l'évolution morphologique des marges arides du ProcheOrient à l'Holocène.

\section{Références}

Ayalon A., Bar Matthews M., Kaufman A. (1999) - Petrography, strontium, barium and uranium concentrations, and strontium and uranium isotope ratios in speleothems as palaeoclimatic proxies: Soreq Cave, Israel. The Holocene, 9, 715-722.

Ballais J.-L. (1984) - Les grandes phases de modification de l'environnement dans les Aurès (Algérie) au cours de la période historique. Bulletin de l'Association de Géographes Français, 499, 73-76.

Ballais J.-L. (1995) - Conquête et dégradation du milieu au Maghreb oriental pendant l'Antiquité et le Moyen Âge. In S. van der Leeuw (Ed.), L'homme et la dégradation de l'environnement. $\mathrm{XV}$ rencontres internationales d'archéologie et d'histoire d'Antibes. Edition APDCA, Sophia Antipolis, 331-344.

Ballais J.-L., Crambes A. (1993) - Morphogenèse holocène et anthropisation sur la montagne Sainte-Victoire. In P. Leveau, M. Provansal (dir.), Archéologie et environnement : de la Sainte-Victoire aux Alpilles. Publications de l'université de Provence, 465-483.

Ballais J.-L., Jorda M., Provansal M, Covo J. (1993) - Morphogenèse holocène sur le périmètre des Alpilles. In P. Leveau, M. Provansal (dir.), Archéologie et environnement : de la Sainte-Victoire aux Alpilles. Publications de l'université de Provence, 65-74. 
Bar-Matthews M., Ayalon A., Kaufman A. (2000) - Timing and hydrological conditions of Sapropels events in the Eastern Mediterranean, as evident from speleothems, Soreq cave, Israel. Chemical Geology, 169, 145-156.

Barker G. (1995) - Land use and environmental degradation in the Biferno Valley (Central Southern Italy) from Prehistoric times to the Present day. In S. van der Leeuw (Ed.), L'homme et la dégradation de l'environnement. $\mathrm{XV}^{\mathrm{e}}$ rencontres internationales d'archéologie et d'histoire d'Antibes. Edition APDCA, Sophia Antipolis, 285-297.

Besançon J. (1994) - Oscillations climatiques et morphogenèse quaternaire au Proche-Orient. Annales de géographie de l'université Saint-Joseph (Beyrouth, Liban), 15, 93-116.

Besançon J., Sanlaville P. (1984) - Terrasses fluviatiles au Proche-Orient. Bulletin de l'Association Française pour l'Étude du Quaternaire, 1-2-3, 186-191.

Besançon J., Sanlaville P. (1985) - Résultats de deux prospections archéologiques effectuées dans la région du Nahr Sajour et sur le haut Euphrate syrien. In P. Sanlaville (éd.) Holocene Settlement in North Syria. BAR International Series, Oxford, British Archaeological Reports,. 7-40.

Besançon J., Delgiovine A., Fontugne M., Lalou C., Sanlaville P., Vaudour J. (1997) - Mise en évidence et datation de phases humides du Pléistocène supérieur dans la région de Palmyre. Paléorient, 23, 5-23.

Brew G., Barazangi M., Al-Maleh A. K., Sawaf T. (2001) - Tectonic and Geologic Evolution of Syria. GeoArabia, 6, 573-615.

Brückner H. (1990) - Changes in the Mediterranean ecosystem during Antiquity. A geomorphological Approach as seen in two Examples. In S. Bottema, G. Entjes-Neiborg and W. van Zeist (Eds.), Man's Role in the Shaping of the Eastern Mediterranean Landscape. Proceedings of the INQUA/BAI, Rotterdam, A.A. Balkema, 127-137.

Bruins H. J. (1994) - Comparative chronology of climate and human history in the Southern Levant from the Late Chalcolithic to the Early Arab Period. In O. Bar-Yosef et R.S. Kra (Eds.), Late Quaternary Chronology And Paleoclimates of The Eastern Mediterranean. Radiocarbon, Tucson-Cambridge (USA), the university of Arizona, Harvard University, 301-314.

Butzer K. W. (1995) - Environmental change in the Near East and Human Impact on the Land. In J. M. Sasson (Ed.), Civilizations of the ancient Near East, vol. 1, New York, 123-151.

Courty M.-A. (1994) - Le cadre paléogéographique des occupations humaines dans le bassin du Haut-Khabour (Syrie du Nord-Est). Premiers résultats. Paléorient, 20, 21-59.

Debelmas J. et Mascle G. (2003) - Les grandes structures géologiques. Paris, Dunod, 312 p.

Deiri W. (1990) - Contribution à l'étude phyto-écologique et de la potentialité pastorale en Syrie aride. Thèse de doctorat de 3e cycle, université des Sciences et Techniques du Languedoc Montpellier II, $238 \mathrm{p}$.

Devillers B. (2003) - Holocene morphogenesis and land use in eastern Mesaoria (Cyprus), preliminary results. In Fouache E. (Ed.), The Mediterranean World Environment and History. Elsevier, Paris, 363-370.

Dufaure J.-J. (1976) - La terrasse holocène d'Olympie et ses équivalents méditerranéens. Bulletin de l'Association de Géographes Français, 433, 85-94.
Dufaure J.-J. (éd.) (1984) - La mobilité des paysages méditerranéens. Hommage à Pierre Birot, Toulouse, $387 \mathrm{p}$.

Enzel Y., Bookman R. (Ken Tor), Sharon D., Gvirtzman H., Dayan U., Ziv B., Steinc M. (2003) - Late Holocene climates of the Near East deduced from Dead Sea level variations and modern regional winter rainfall, Quaternary Research, 60, 263273.

Fouache E., (1999) - L'Alluvionnement historique en Grèce occidentale et au Péloponnèse : géomorphologie, archéologie, histoire. Athènes, Bulletin de correspondance hellénique 35, $235 \mathrm{p}$.

Geyer B. (1999) - Aridité et sociétés au Proche-Orient, une problématique géo-archéologique. Travail présenté pour l'obtention de l'Habilitation à diriger des recherches, université Lumière Lyon, 168 p.

Geyer B., Lefort J. (éds.) (2003) - La Bithynie au Moyen Âge. Réalités Byzantines 9, Édition P. Lethielleux, Paris, 567 p.

Geyer B., Sanlaville P. (1991) - Signification et chronologie des terrasses holocènes du bassin syrien de l'Euphrate. Physio-Géo, 22-23, 101-106.

Geyer B., Besançon J. (1997) - Environnement et occupation du sol dans la vallée de l'Euphrate syrien durant le Néolithique et le Chalcolithique. Paléorient, 22, 5-15.

Issar A. S., Govrin Y., Geyh M. A., Wakshal E., Wolf M. (1991) - Climate changes during the upper Holocene in Israel. Israel Earth Science, 40, 219-223.

Jaubert R., Debaine F., Besançon J., Al-Dbiyat M., Geyer B., Gintzburger G., Traboulsi M. (1999) - Utilisation du territoire et couvert végétal. Régions arides et semi-arides des provinces d'Alep et de Hama (Syrie). Cahiers de la recherche Hors Série, GREMMO, Lyon, 103 p.

Laborde J.-P., Traboulsi M. (2001) - Cartographie automatique des précipitations : applications aux précipitations annuelles du Moyen-Orient. Publications de l'Association Internationale de Climatologie, 14, 296-303.

Leroux M. (1993) - The Mobile Polar High: a new concept explaining present mechanism of meridional air-mass and energy exchanges and global propagation of palaeoclimatic changes. Global and planetary change, 7, 69-93.

Leroux M. (1996) - La dynamique du temps et du climat. Paris, Masson, $310 \mathrm{p}$.

Neboit R. (1984) - Érosion des sols et colonisation grecque en Sicile et en Grande Grèce. Bulletin de l'Association de Géographes Français, 499, 5-13.

Neboit R. (1991) - L'homme et l'érosion : l'érosion des sols dans le monde. Clermont-Ferrand, publications de la faculté des Lettres, université Blaise-Pascal, 269 p.

Neboit-Guilhot R. (2003) - Le témoignage ambigu des terrasses historiques de piémont en Italie méridionale. In É. Fouache (Ed.) The Mediterranean World Environment and History. Elsevier, Paris, 17-23.

Ponikarov V.P., Protasevitch L. N., Maximov A. A. (1966) - The geological map of Syria. Échelle 1:200 000, feuilles J-37-I, II (Halab, Antakya) et I-37-XX (Salamiye), avec notices explicatives, Syrian Arab Republic.

Rosen A. M. (1997) - The geoarchaeology of Holocene environments and landuse at Kazane Höyük, SE Turkey. Geoarchaeology, 12, 395-416. 
Rösner U., Schäbitz F. (1991) - Palynological and sedimentological evidence for the historic environment of Khatouniye, eastern Syrian Djezire. Paléorient, 17, 77-88.

Rossignol-Strick M. (1999) - The Holocene climatic optimum and pollen records of sapropel 1 in Eastern Mediterranean, 9 000-6 000 BP. Quaternary Science Review, 18, 515-530.

Sanlaville P. (1977) - Étude géomorphologique de la région littorale du Liban. Beyrouth, université libanaise, 2 vol., 860 p.

Sanlaville P. (1996) - Changements climatiques dans la région levantine à la fin du Pléistocène supérieur et au début de l'Holocène. Leur relation avec l'évolution des sociétés humaines. Paléorient, 22, 7-30.

Sanlaville P. (1997) - Les changements dans l'environnement au Moyen-Orient de 20000 à 6000 BP. Paléorient, 23, 249262.

Sanlaville P. (dir.) (1979) - Quaternaire et préhistoire du Nahr elKébir septentrional : les débuts de l'occupation humaine dans la Syrie du Nord et au Levant. Collection de la Maison de l'Orient Méditerranéen $n^{\circ}$ 9, Série géographique et préhistorique $\mathrm{n}^{\circ} 1$. Paris, CNRS, $161 \mathrm{p}$.

Schilman B., Bar-Matthews M., Almogi-Labin A., Luz B (2001) - Global climate instability reflected by Eastern Mediterranean marine records during the late Holocene. Palaeogeography, Palaeoclimatology, Palaeoecology, 176, 157-176.

Traboulsi M. (1981) - Le climat de la Syrie : exemple de dégradation vers l'aride du climat méditerranéen. Thèse de $3^{\mathrm{e}}$ cycle en géographie, université Lumière Lyon 2, 477 p.

Traboulsi M. (1991) - La variabilité des précipitations dans le désert Syrien. Méditerranée, 4, 47-54.

Traboulsi M. (2004) - Les précipitations au Proche-Orient. Variabilité spatio-temporelle et relation avec la dynamique de l'atmosphère (1960-61/1989-90). Thèse de doctorat, université de Bourgogne, 233 p.

Vita-Finzi C. (1969) - Mediterranean Valleys. Geological changes in historical times, Cambridge University Press, 140 p.

Wilkinson T. J. (1999) - Holocene valley fills of southern Turkey and northwestern Syria: recent geoarchaeological contributions. Quaternary Science Review, 18, 555-571.

Yakir D., Issar A., Gat J., Adar E., Trimborn P., Lipp J. (1994) $-{ }^{13} \mathrm{C}$ and ${ }^{18} \mathrm{O}$ of wood from the Roman siege rampart in Masada, Israel (AD 70-73): Evidence for a less arid climate for the region. Geochimica et Cosmochimica Acta, 58, 3535-3539.

Article reçu le 20 novembre 2004, accepté le 8 novembre 2006. 
